\title{
Dissipative Solutions and Semiflow Selection for the Complete Euler System
}

\author{
Dominic Breit ${ }^{1}$, Eduard Feireisl ${ }^{2,3}$, Martina Hofmanová ${ }^{4}$ \\ 1 Department of Mathematics, Heriot-Watt University, Riccarton Edinburgh EH14 4AS, UK. \\ E-mail: d.breit@hw.ac.uk \\ 2 Institute of Mathematics AS CR, Žitná 25, 11567 Praha 1, Czech Republic \\ 3 Institute of Mathematics, TU Berlin, Strasse des 17.Juni, Berlin, Germany. \\ E-mail: feireisl@math.cas.cz \\ 4 Fakultät für Mathematik, Universität Bielefeld, 33501 Bielefeld, Germany. \\ E-mail: hofmanova@math.uni-bielefeld.de
}

Received: 29 March 2019 / Accepted: 23 October 2019

Published online: 13 January 2020 - (C) The Author(s) 2020

\begin{abstract}
To circumvent the ill-posedness issues present in various models of continuum fluid mechanics, we present a dynamical systems approach aiming at the selection of physically relevant solutions. Even under the presence of infinitely many solutions to the full Euler system describing the motion of a compressible inviscid fluid, our approach permits to select a system of solutions (one trajectory for every initial condition) satisfying the classical semiflow property. Moreover, the selection respects the well accepted admissibility criteria for physical solutions, namely, maximization of the entropy production rate and the weak-strong uniqueness principle. Consequently, strong solutions are always selected whenever they exist and stationary states are stable and included in the selection as well. To this end, we introduce a notion of dissipative solution, which is given by a triple of density, momentum and total entropy defined as expectations of a suitable measure-valued solution.
\end{abstract}

\section{Contents}

1. Introduction . . . . . . . . . . . . . . . . . . . 1472

1.1 Admissibility criteria . . . . . . . . . . . . . . . . . . . 1473

1.2 Semiflow solutions . . . . . . . . . . . . . . . . . . . 1475

1.3 Selection procedure . . . . . . . . . . . . . . . . . 1477

2. Dynamical Systems Approach . . . . . . . . . . . . . . . . . . 1477

2.1 Trajectory space . . . . . . . . . . . . . . . . . . . . . . . 1478

2.2 Solution space . . . . . . . . . . . . . . . . . . . . . . . 1478

3. Dissipative Solutions . . . . . . . . . . . . . . . . . . . . . . . . . . . . . . . . . . . . . . . . . . . . . . . . .

3.1 Phase space . . . . . . . . . . . . . . . . . . . 1479

The research of E.F. leading to these results has received funding from the Czech Sciences Foundation (GAČR), Grant Agreement 18-05974S. The Institute of Mathematics of the Academy of Sciences of the Czech Republic is supported by RVO:67985840. M.H. gratefully acknowledges the financial support by the German Science Foundation DFG via the Collaborative Research Center SFB1283. 
3.2 Dissipative measure-valued solutions . . . . . . . . . . . . . . . . 1481

3.3 Dissipative solution . . . . . . . . . . . . . . . . . . . . . 1485

3.4 Sequential stability . . . . . . . . . . . . . . . . . . . . . . . 1486

3.5 Existence of dissipative solutions . . . . . . . . . . . . . . . . . . . . 1489

4. Semiflow Selection . . . . . . . . . . . . . . . . . . . . . 1491

5. Concluding Discussion . . . . . . . . . . . . . . . . . . . 1493

5.1 Stability of equilibrium states . . . . . . . . . . . . . . . . . . . . . 1493

5.2 Dafermos' admissibility criteria . . . . . . . . . . . . . . . . . 1494

References . . . . . . . . . . . . . . . . . . . . . . . 1497

\section{Introduction}

The Euler system describing the motion of a general compressible inviscid fluid represents one of the basic models in the framework of continuum fluid mechanics. The unknown fields are the fluid density $\varrho=\varrho(t, x)$, the momentum $\mathbf{m}=\mathbf{m}(t, x)$, and the energy $\mathcal{E}=\mathcal{E}(t, x)$ satisfying the system of partial differential equations:

$$
\begin{aligned}
\partial_{t} \varrho+\operatorname{div}_{x} \mathbf{m} & =0, \\
\partial_{t} \mathbf{m}+\operatorname{div}_{x}\left(\frac{\mathbf{m} \otimes \mathbf{m}}{\varrho}\right)+\nabla_{x} p & =0, \\
\partial_{t} \mathcal{E}+\operatorname{div}_{x}\left[(\mathcal{E}+p) \frac{\mathbf{m}}{\varrho}\right] & =0 .
\end{aligned}
$$

Writing the energy as a sum of its kinetic and internal component,

$$
\mathcal{E}=\frac{1}{2} \frac{|\mathbf{m}|^{2}}{\varrho}+\varrho e
$$

we suppose that the pressure $p$ and the internal energy $e$ satisfy the caloric equation of state in the form

$$
(\gamma-1) \varrho e=p, \text { where } \gamma>1 \text { is the adiabatic constant. }
$$

In addition, we introduce the absolute temperature $\vartheta$ through the Boyle-Mariotte thermal equation of state:

$$
p=\varrho \vartheta \text { yielding } e=c_{v} \vartheta, c_{v}=\frac{1}{\gamma-1} .
$$

Supposing that the fluid occupies a bounded spatial domain $\Omega \subset R^{N}, N=1,2,3$ we impose the impermeability boundary condition

$$
\left.\mathbf{m} \cdot \mathbf{n}\right|_{\partial \Omega}=0 .
$$

Finally, the initial state of the fluid is given through the initial conditions

$$
\varrho(0, \cdot)=\varrho_{0}, \mathbf{m}(0, \cdot)=\mathbf{m}_{0}, \mathcal{E}(0, \cdot)=\mathcal{E}_{0} .
$$

The Second law of thermodynamics is enforced through the entropy balance equation

$$
\partial_{t}(\varrho s)+\operatorname{div}_{x}(s \mathbf{m})=0 \text { or } \partial_{t} s+\left(\frac{\mathbf{m}}{\varrho}\right) \cdot \nabla_{x} s=0,
$$


where the entropy $s$ is given as

$$
s(\varrho, \vartheta)=\log \left(\vartheta^{c_{v}}\right)-\log (\varrho) .
$$

There is a vast amount of literature dedicated to the mathematical theory of the Euler system. In particular, it is known that the initial-value problem is well posed locally in time in the class of smooth solutions, see e.g. the monograph by Majda [17] or the more recent treatment by Benzoni-Gavage and Serre [1]. Smooth solutions, however, develop singularities in a finite time for a fairly general class of initial data, see e.g. Smoller [19]. Thus, if the Euler system is accepted as an adequate description of the fluid motion in a long run, a concept of generalized solutions is needed.

The modern theory of partial differential equations is based on the concept of weak solution, where derivatives are understood in the sense of distributions. This gives rise to a large class of objects in which uniqueness might be lost. Several admissibility criteria have been proposed to select the physically relevant weak solution, among which the entropy inequality

$$
\partial_{t}(\varrho s)+\operatorname{div}_{x}(s \mathbf{m}) \geq 0
$$

reflecting the Second law of thermodynamics. The recent adaptation of the method of convex integration, developed in the context of incompressible fluids by De Lellis and Székelyhidi [10], gave rise to numerous examples of ill-posedness also in the class of compressible fluids, see Chiodaroli, De Lellis, Kreml [6], Chiodaroli and Kreml [7] , Chiodaroli et al. [8], among others. In particular, it was shown in [12] that the Euler system (1.1)-(1.5) is ill-posed, specifically it admits infinitely many weak solutions for a large class of initial data. Moreover, these solutions satisfy the entropy inequality (1.8). In addition, examples of regular initial data producing infinitely many weak solutions in the long run have been also obtained in [12].

1.1. Admissibility criteria. In view of these facts, more refined admissibility criteria are needed in order to select the physically relevant solutions. Dafermos [9] proposed a selection criterion based on maximality of the total entropy. We formulate it in a slightly stronger form, avoiding the issue of existence of right-sided time derivatives. Specifically, suppose that $\left[\varrho_{i}, \mathbf{m}_{i}, \mathcal{E}_{i}\right], i=1,2$ are two solutions of the Euler system (1.1)-(1.5) with the associated total entropies

$$
S_{i}=\varrho_{i} s\left(\varrho_{i}, \mathbf{m}_{i}, \mathcal{E}_{i}\right), i=1,2 .
$$

We say that

$$
\left[\varrho_{1}, \mathbf{m}_{1}, \mathcal{E}_{1}\right] \succ \mathcal{D}\left[\varrho_{2}, \mathbf{m}_{2}, \mathcal{E}_{2}\right]
$$

if:

- there exists $\tau \geq 0$ such that

$$
\left[\varrho_{1}(t, \cdot), \mathbf{m}_{1}(t, \cdot), \mathcal{E}_{1}(t, \cdot)\right]=\left[\varrho_{2}(t, \cdot), \mathbf{m}_{2}(t, \cdot), \mathcal{E}_{2}(t, \cdot)\right] \text { for any } t \in[0, \tau] ;
$$

- there exists $\delta>0$ such that

$$
\int_{\Omega} S_{1}(t+, x) \mathrm{d} x \geq \int_{\Omega} S_{2}(t+, x) \mathrm{d} x \text { for all } t \in(\tau, \tau+\delta) .
$$


A weak solution $[\varrho, \mathbf{m}, \mathcal{E}]$ is called maximal (admissible) if it is maximal with respect to the relation $\succ_{\mathcal{D}}$.

A modification of this criterion was further investigated in the context of the barotropic Euler system in [11]. However, in this case it is rather the total energy that shall be minimized, according to the principle of maximal energy dissipation. The criterion of [11] translated to our setting of the full Euler system, leads to a weaker version of the condition (1.9). Namely, we require that there exists a sequence $\left(\tau_{n}\right)_{n}, \tau_{n}>\tau, \tau_{n} \rightarrow \tau$ such that

$$
\int_{\Omega} S_{1}\left(\tau_{n}+, x\right) \mathrm{d} x \geq \int_{\Omega} S_{2}\left(\tau_{n}+, x\right) \mathrm{d} x \text { for all } n \in \mathbb{N} .
$$

It was shown in [11] that the solutions constructed there by the method of convex integration do not fulfil the corresponding criterion of maximal energy dissipation, suggesting that such a criterion shall be retained in order to exclude nonphysical solutions.

To compare these two criteria, let us denote by $\succ_{\mathcal{F}}$ the partial ordering induced by (1.10). The following holds true: If a solution is maximal with respect to $\succ_{\mathcal{F}}$ then it is also maximal with respect to $\succ_{\mathcal{D}}$. This may seem surprising at first sight since (1.9) is obviously a stronger condition than (1.10). But it is exactly for this reason why the implication of maximality is valid. More precisely, the weaker condition (1.10) allows to compare more solutions, for instance also those that oscillate around each other and that are therefore not comparable by the condition (1.9).

An alternative criterion enforcing the Second law of thermodynamics is maximility of the global entropy production proposed in [3]. In accordance with the Schwartz representation principle, the inequality in the entropy balance (1.8) can be interpreted as

$$
\partial_{t} S+\operatorname{div}_{x}\left(S \frac{\mathbf{m}}{\varrho}\right)=\Sigma, S=\varrho s,
$$

where $\Sigma$ is a non-negative Borel measure on $[0, \infty) \times \bar{\Omega}$. Similarly to the above, we say that

$$
\begin{gathered}
{\left[\varrho_{1}, \mathbf{m}_{1}, \mathcal{E}_{1}\right] \succ\left[\varrho_{2}, \mathbf{m}_{2}, \mathcal{E}_{2}\right]} \\
\Leftrightarrow \Sigma_{1} \geq \Sigma_{2} \text { on any compact subset of }[0, \infty) \times \bar{\Omega} .
\end{gathered}
$$

It was shown in [3] that maximal solutions with respect to the relation $\succ$ exist for the full Euler system under rather general hypotheses on the data as well as the constitutive relations.

Despite these efforts, however, none of the above selection criteria proved sufficient to guarantee the desired well-posedness result. The major open question therefore remains: how can physically relevant solutions be distinguished from the nonphysical ones? The aim of the present paper is to take into account another physical property of an evolution system, namely, the so-called semiflow property: starting the system at time 0 , letting it run to time $s>0$ and then restarting and continue for the time $t>0$, the state of the system at the final time $s+t$ should be the same as if the system runs directly from 0 to $s+t$. If uniqueness holds, the semiflow property immediately follows. However, for systems where uniqueness is unknown or not valid, it is generally not clear whether such a semiflow even exists. 
1.2. Semiflow solutions. On the following pages we show how to approach this problem and we construct a solution semiflow to the complete Euler system (1.1)-(1.4). In particular, this leads to a well-defined dynamical system associated to (1.1)-(1.4), which depends in a measurable way on the initial condition. To this end, it is in the first place necessary to identify the correct phase variables of the system together with a suitable notion of solution. Even though the system (1.1) describes the evolution of the density, momentum and energy, it turns out to be beneficial to replace the energy by the total entropy. In other words, the state of the fluid at a given time $t \geq 0$ will be determined by the values of three phase variables,

the density $\varrho(t, \cdot)$, the momentum $\mathbf{m}(t, \cdot)$, the total entropy $S(t, \cdot)=\varrho s(t, \cdot)$, interpreted through their spatial integral means as quantities in suitable abstract function spaces.

The reason why we prefer the entropy $S$ instead of the energy $\mathcal{E}$ is the lack of suitable a priori bounds for the latter. The integral means

$$
t \mapsto \int_{\Omega} \varrho(t, \cdot) \varphi \mathrm{d} x, t \mapsto \int_{\Omega} \mathbf{m}(t, \cdot) \cdot \varphi \mathrm{d} x
$$

will be continuous for $t \in[0, \infty)$ for any smooth $\varphi, \varphi$, while

$$
t \mapsto \int_{\Omega} S(t, \cdot) \varphi \mathrm{d} x \in \mathrm{BV}_{\text {loc }}[0, \infty) ;
$$

whence the limits

$$
\int_{\Omega} S(t+, \cdot) \varphi \mathrm{d} x, \int_{\Omega} S(t-, \cdot) \varphi \mathrm{d} x \text { are well defined for } t \in[0, \infty)
$$

with the convention

$$
S(0-)=S_{0}
$$

Hence, another difficulty regarding the classical theory of dynamical systems stems from the fact that solutions are not continuous in time, which in particular holds here for the total entropy. We overcome this issue by replacing continuity by existence of one-sided limits at every time $t \geq 0$.

As the next step, we shall determine what input information on the initial state of the system is necessary. Apart from the initial state for the density, momentum and total entropy, we shall be also given the total energy $E_{0}$ which is a constant of the motion and provides various bounds for all the corresponding quantitities. Given the initial state of the system $\mathbb{U}_{0}=\left(\mathbf{U}_{0}, E_{0}\right)$, where

$$
\begin{aligned}
& \mathbf{U}_{0}=\left[\varrho_{0}, \mathbf{m}_{0}, S_{0}\right], \text { together with the total energy } \\
& E_{0} \geq \int_{\Omega}\left[\frac{\left|\mathbf{m}_{0}\right|^{2}}{\varrho_{0}}+\varrho_{0} e\left(\varrho_{0}, S_{0}\right)\right] \mathrm{d} x,
\end{aligned}
$$

we then identify in a unique way the state of the system at the time $t \geq 0$,

$$
\begin{aligned}
& \mathbb{U}\left[t ;\left(\mathbf{U}_{0}, E_{0}\right)\right]=\left([\varrho(t, \cdot), \mathbf{m}(t, \cdot), S(t-, \cdot)], E_{0}\right), \\
& E_{0} \geq \int_{\Omega}\left[\frac{|\mathbf{m}(t, \cdot)|^{2}}{\varrho(t, \cdot)}+\varrho(t, \cdot) e(\varrho(t, \cdot), S(t \pm, \cdot))\right] \mathrm{d} x .
\end{aligned}
$$

In addition, the mapping $t \mapsto \mathbb{U}\left[t ;\left(\mathbf{U}_{0}, E_{0}\right)\right]$ will enjoy the semiflow property: 


$$
\mathbb{U}\left[0 ;\left(\mathbf{U}_{0}, E_{0}\right)\right]=\left(\mathbf{U}_{0}, E_{0}\right)
$$

$$
\mathbb{U}\left[t_{1}+t_{2} ;\left(\mathbf{U}_{0}, E_{0}\right)\right]=\mathbb{U}\left[t_{2} ; \mathbb{U}\left[t_{1},\left(\mathbf{U}_{0}, E_{0}\right)\right]\right] \text { for any } t_{1}, t_{2} \geq 0 .
$$

In particular, the state of the system at a time $t \geq 0$ is uniquely determined in terms of the initial data $\left[\varrho_{0}, \mathbf{m}_{0}, S_{0}\right]$ and the initial energy $E_{0}$.

The trajectories

$$
t \mapsto \mathbb{U}\left[t ;\left(\mathbf{U}_{0}, E_{0}\right)\right]=\left([\varrho(t, \cdot), \mathbf{m}(t, \cdot), S(t-, \cdot)], E_{0}\right)
$$

represent a generalized—dissipative—solution to the Euler system (1.1)-(1.5), which we introduce in Sect. 3. It will be shown that they comply with the following stipulations:

- Weak-strong uniqueness. Suppose that the Euler system (1.1)-(1.5) admits a classical solution $[\varrho, \mathbf{m}, \mathcal{E}]$ on a time interval $\left[0, T_{\max }\right)$,

$$
E_{0}=\int_{\Omega} \mathcal{E}_{0} \mathrm{~d} x
$$

Then for $\mathbf{U}_{0}=\left[\varrho_{0}, \mathbf{m}_{0}, S\left(\varrho_{0}, \mathbf{m}_{0}, \mathcal{E}_{0}\right)\right]$ we have

$$
\mathbb{U}\left[t ;\left(\mathbf{U}_{0}, E_{0}\right)\right]=\left([\varrho(t, \cdot), \mathbf{m}(t, \cdot), S(\varrho(t, \cdot), \mathbf{m}(t, \cdot), \mathcal{E}(t, \cdot))], E_{0}\right)
$$

for all $t \in\left[0, T_{\max }\right)$.

- Maximal entropy production. Suppose that

$$
\left(\left[\varrho_{1}, \mathbf{m}_{1}, S_{1}\right], E_{0}\right)=\mathbb{U}\left[\cdot ;\left(\mathbf{U}_{0}, E_{0}\right)\right]
$$

and that $\left(\left[\varrho_{2}, \mathbf{m}_{2}, S_{2}\right], E_{0}\right)$ is another dissipative solution starting from the same initial data $\left(\mathbf{U}_{0}, E_{0}\right)$ and such that

$$
\sigma_{1}(t):=\int_{\Omega}\left(S_{1}(t)-S_{0}\right) \mathrm{d} x \leq \sigma_{2}(t):=\int_{\Omega}\left(S_{2}(t)-S_{0}\right) \mathrm{d} x \text { on }[0, \infty),
$$

where $\sigma_{i}$ is the entropy production rate associated to $\left[\varrho_{i}, \mathbf{m}_{i}, S_{i}\right], i=1,2$, respectively.

Then

$$
\sigma_{1}=\sigma_{2}
$$

- Stability of stationary states. Let $\varrho=\bar{\varrho}>0, \mathbf{m}=0, \overline{\mathcal{E}}=\frac{1}{|\Omega|} E_{0}$ be a stationary solution of the Euler system (1.1). Suppose that the system reaches the equilibrium state at some time $T \geq 0$, i.e.

$$
\mathbb{U}\left[T ;\left(\left[\varrho_{0}, \mathbf{m}_{0}, S_{0}\right] ; E_{0}\right)\right]=\left([\bar{\varrho}, 0, S(\bar{\varrho}, 0, \overline{\mathcal{E}})], E_{0}\right) .
$$

Then

$$
\mathbb{U}\left[t ;\left(\left[\varrho_{0}, \mathbf{m}_{0}, S_{0}\right] ; E_{0}\right)\right]=\left([\bar{\varrho}, 0, S(\bar{\varrho}, 0, \overline{\mathcal{E}})], E_{0}\right)
$$

for all $t \geq T$. 
1.3. Selection procedure. The semiflow $\mathbb{U}$ will be constructed by means of the selection procedure originally proposed in the context of stochastic Markov processes by Krylov [16]. The method was later adapted to deterministic evolutionary problems with time continuous solutions by Cardona and Kapitanski [5]. We have developed a similar approach to the isentropic Euler system in [2], where we also relaxed the continuity assumption on the trajectories. We refer the reader to Sect. 2 for details of the abstract formulation.

In Sect. 3, we specify a class of generalized solutions to the Euler system (1.1)(1.5) termed dissipative solutions. They are, loosely speaking, the expected values of suitable measure-valued solutions as introduced in [4] but with an additional refinement regarding the associated concentration defect measures.

Applying carefully the selection procedure of $[2,5,16]$, we obtain the desired semiflow solution in Sect. 4. The paper is concluded by a discussion of further extensions in Sect. 5. In particular, in Sect. 5.2 we compare our construction to the two versions of Dafermos' criterium introduced in Sect. 1.1.

\section{Dynamical Systems Approach}

We start by adapting the general approach of $[5,16]$ to problems with discontinuous solutions paths, see also [2]. Suppose that the state of a physical system at each instant $t \geq 0$ is characterized by an abstract vector $\mathbb{U}(t) \in \mathcal{S}$ ranging in a suitable phase space $\mathcal{S}$. In our setting, the state space will be a separable Hilbert space or a Polish topological space.

Consider a mapping

$$
\mathbb{U}:[0, \infty) \times \mathcal{S} \rightarrow \mathcal{S},\left[t, \mathbb{U}_{0}\right] \mapsto \mathbb{U}\left[t ; \mathbb{U}_{0}\right]
$$

meaning $\mathbb{U}\left[t ; \mathbb{U}_{0}\right]$ is the state of the system emanating from the initial state $\mathbb{U}_{0}$ at a time $t \geq 0$.

Definition 2.1 (Semiflow). We say that a mapping $\mathbb{U}:[0, \infty) \times \mathcal{S} \rightarrow \mathcal{S}$ is a semiflow if:

(a) The flow starts at the initial datum, that is

$$
\mathbb{U}\left[0, \mathbb{U}_{0}\right]=\mathbb{U}_{0} \text { for any } \mathbb{U}_{0} \in \mathcal{S} \text {. }
$$

(b) It has the semigroup property, that is

$$
\mathbb{U}\left[t_{1}+t_{2}, \mathbb{U}_{0}\right]=\mathbb{U}\left[t_{2}, \mathbb{U}\left[t_{1}, \mathbb{U}_{0}\right]\right] \text { for any } \mathbb{U}_{0} \in \mathcal{S}, t_{1}, t_{2} \geq 0 .
$$

In the classical theory of dynamical systems, continuity of the semiflow $\mathbb{U}$ is required both in $t$ and in $\mathbb{U}_{0}$. In view of the issues discussed in the previous section, we develop a generalized theory, where continuity in time is relaxed to the existence of the one-sided limits at $t \pm$, while continuity in $\mathbb{U}_{0}$ is replaced by measurability with respect to Borel sets generated by suitable topologies. 
2.1. Trajectory space. The trajectories space can be defined as $\mathcal{T}=\mathrm{BV}_{\text {loc }}([0, \infty) ; \mathcal{S})$. For trajectories we can define the following operations:

- Time shift. For $\xi \in \mathcal{T}$ and $T>0$ we set

$$
\mathfrak{S}_{T}[\xi](t \pm)=\xi((T+t) \pm) \text { for } t \in[0, \infty) .
$$

- Continuation. For $\xi^{1}, \xi^{2} \in \mathcal{T}$ and $T>0$ we set

$$
\xi^{1} \cup_{T} \xi^{2}(t \pm)= \begin{cases}\xi^{1}(t \pm) \text { if } 0<t<T, & \xi^{1} \cup_{T} \xi^{2}(T-)=\xi^{1}(T-), \\ \xi^{2}((t-T) \pm) \text { for } t>T, & \xi^{1} \cup_{T} \xi^{2}(T+)=\xi^{2}(0+) .\end{cases}
$$

2.2. Solution space. The solution space can be loosely described as the family of all solutions of a given system of equations emanating from a fixed initial datum in $\mathcal{S}$. As such the solution operator $\mathcal{U}$ can be understood as a mapping

$$
\mathcal{U}: \mathcal{S} \rightarrow 2^{\mathcal{T}}, \mathcal{U}\left[\mathbb{U}_{0}\right] \subset \mathcal{T}, \mathbb{U}\left[\mathbb{U}_{0}\right](0-)=\mathbb{U}_{0} \text { for all } \mathbb{U} \in \mathcal{U}\left[\mathbb{U}_{0}\right]
$$

Definition 2.2 (Solution operator). We say that a mapping $\mathcal{U}: \mathcal{S} \rightarrow 2^{\mathcal{T}}$ is a solution operator if the following properties are satisfied:

[A1] Existence, compactness. For each $\mathbb{U}_{0} \in \mathcal{S}$ the set $\mathcal{U}\left[\mathbb{U}_{0}\right]$ is a non-empty bounded subset of $\mathrm{BV}_{\text {loc }}([0, \infty) ; \mathcal{S})$, compact in the topology of $L_{\text {loc }}^{1}([0, \infty) ; \mathcal{S})$,

$$
\mathbb{U}\left[\mathbb{U}_{0}\right](0-)=\mathbb{U}_{0} \text { for any } \mathbb{U} \in \mathcal{U}\left[\mathbb{U}_{0}\right] .
$$

[A2] Measurability. The set valued mapping

$$
\mathcal{U}: \mathbb{U}_{0} \in \mathcal{S} \mapsto \mathcal{U}\left[\mathbb{U}_{0}\right] \in 2^{\mathcal{T}}
$$

is Borel measurable; where $2^{\mathcal{T}}$ is endowed with the Hausdorff topology defined on compact subsets of the metric space $L_{\text {loc }}^{1}([0, \infty) ; \mathcal{S})$.

[A3] Shift invariance. For any $\mathbb{U} \in \mathcal{U}\left[\mathbb{U}_{0}\right]$ and any $T>0$, we have

$$
\mathfrak{S}_{T}[\mathbb{U}] \in \mathcal{U}[\mathbb{U}(T-)] .
$$

[A4] Continuation. For any $\mathbb{U}^{1} \in \mathcal{U}\left[\mathbb{U}_{0}\right], T>0$, and $\mathbb{U}^{2} \in \mathcal{U}\left[\mathbb{U}^{1}(T-)\right]$, we have

$$
\mathbb{U}^{1} \cup_{T} \mathbb{U}^{2} \in \mathcal{U}\left[\mathbb{U}_{0}\right]
$$

A version of the following result for the case of $\mathcal{T}$ being a set of continuous trajectories with values in a Polish space was proved by Cardona and Kapitanski [5]. However, for applications in fluid dynamics, where time continuity of the energy or entropy is not valid, a suitable modification is necessary which was proved in [2, Section 5].

Proposition 2.3. Let $\mathcal{S}$ be a Polish space and let $\mathcal{T}=\mathrm{BV}_{\mathrm{loc}}([0, \infty) ; \mathcal{S})$. Let $\mathcal{U}: \mathcal{S} \rightarrow$ $2^{\mathcal{T}}$, be a solution operator enjoying the properties [A1]-[A4] from Definition 2.2.

Then $\mathcal{U}$ admits a measurable semiflow selection. Specifically, for any $\mathbb{U}_{0} \in \mathcal{S}$ there exists a single trajectory

$$
\mathbb{U} \in \mathcal{U}\left[\mathbb{U}_{0}\right], \mathbb{U}: \mathcal{S} \rightarrow \mathcal{T} \subset L_{\mathrm{loc}}^{1}([0, \infty) ; \mathcal{S}) \text { Borel measurable },
$$


such that

$$
\mathbb{U}:\left[t, \mathbb{U}_{0}\right] \in[0, \infty) \times \mathcal{S} \mapsto \mathbb{U}\left[\mathbb{U}_{0}\right](t-), t>0, \mathbb{U}(0-)=\mathbb{U}_{0}
$$

is a semiflow in the sense of Definition 2.1.

In addition, if $\beta: \mathcal{S} \rightarrow R$ is a bounded continuous function and $\lambda>0$, the selection can be chosen to satisfy

$$
\int_{0}^{\infty} \exp (-\lambda t) \beta\left(\mathbb{U}\left[t ; \mathbb{U}_{0}\right]\right) \mathrm{d} t \leq \int_{0}^{\infty} \exp (-\lambda t) \beta(\mathbb{V}(t)) \mathrm{d} t \quad \forall \mathbb{V} \in \mathcal{U}\left[\mathbb{U}_{0}\right]
$$

We recall that the selection procedure of Proposition 2.3 relies on a subsequent minimization (or alternatively maximization) of a sequence of suitable continuous functionals, as e.g. the functional in (2.1), over the set of all solutions. The functionals are chosen in a way to separate points of the trajectory space $\mathcal{T}$. For this purpose the Laplace transform with respect to the time variable proved to be beneficial. In general, the selection depends on the particular choice of a sequence $\left(\lambda_{n}\right)_{n \in \mathbb{N}}$ representing the points where the Laplace transform is evaluated. The functional in (2.1) shall then be chosen as the first one to minimize/maximize. In our application to the complete Euler system in the next section we chose (2.1) in order to maximize the entropy production rate.

In the remaining part of the paper, we identify a suitable solution operator $\mathcal{U}$ associated to the Euler system that complies with the hypotheses of Proposition 2.3. In Sect. 5, we present further discussion on the question of optimality of the choice of the functionals and in particular the relation to the Dafermos criterion discussed in the introduction.

\section{Dissipative Solutions}

Our first goal in this section is to identify a suitable class of generalized solutions to the Euler system (1.1)-(1.5). We start by introducing the basic state variables, then we recall the (slightly modified) notion of dissipative measure-valued solutions from [4]. This leads us to our notion of dissipative solution which we introduce in Sect. 3.3. Eventually, we study stability of dissipative solutions as well as their existence. The semiflow is then constructed in Sect. 4.

3.1. Phase space. In what follows, we plan to work with the state variables $\varrho, \mathbf{m}$, and the total entropy $S=\varrho s$. In accordance with hypotheses (1.2) and (1.3) (compare also (1.7) with) the pressure $p$ and the internal energy $e$ can be written in the form

$$
p=p(\varrho, S)=\varrho^{\gamma} \exp \left(\frac{S}{c_{v} \varrho}\right), e=e(\varrho, S)=\frac{1}{\gamma-1} \varrho^{\gamma-1} \exp \left(\frac{S}{c_{v} \varrho}\right)
$$

Lemma 3.1. The mapping

$$
(\varrho, S) \mapsto p(\varrho, S), \varrho>0, S \in R
$$

is strictly convex. 
Proof. This is a matter of direct computation of the Hessian matrix. We have

$$
\begin{aligned}
& \frac{\partial p(\varrho, S)}{\partial \varrho}=\gamma \varrho^{\gamma-1} \exp \left(\frac{S}{c_{v} \varrho}\right)-\frac{S}{c_{v}} \varrho^{\gamma-2} \exp \left(\frac{S}{c_{v} \varrho}\right), \\
& \frac{\partial p(\varrho, S)}{\partial S}=\frac{1}{c_{v}} \varrho^{\gamma-1} \exp \left(\frac{S}{c_{v} \varrho}\right),
\end{aligned}
$$

and

$$
\begin{aligned}
\frac{\partial^{2} p(\varrho, S)}{\partial \varrho^{2}} & =\left[(\gamma-1) \varrho^{2}+\left((\gamma-1) \varrho-\frac{S}{c_{v}}\right)^{2}\right] \varrho^{\gamma-4} \exp \left(\frac{S}{c_{v} \varrho}\right), \\
\frac{\partial^{2} p(\varrho, S)}{\partial S^{2}} & =\frac{1}{c_{v}^{2}} \varrho^{\gamma-2} \exp \left(\frac{S}{c_{v} \varrho}\right)=\frac{1}{c_{v}^{2}} \varrho^{2} \varrho^{\gamma-4} \exp \left(\frac{S}{c_{v} \varrho}\right), \\
\frac{\partial^{2} p(\varrho, S)}{\partial \varrho \partial S} & =\frac{1}{c_{v}}(\gamma-1) \varrho^{\gamma-2} \exp \left(\frac{S}{c_{v} \varrho}\right)-\frac{S}{c_{v}^{2}} \varrho^{\gamma-3} \exp \left(\frac{S}{c_{v} \varrho}\right) \\
& =\left[\frac{1}{c_{v}}(\gamma-1) \varrho^{2}-\frac{S}{c_{v}^{2}} \varrho\right] \varrho^{\gamma-4} \exp \left(\frac{S}{c_{v} \varrho}\right) .
\end{aligned}
$$

Obviously the Hessian matrix has positive trace, while its determinant reads

$$
\begin{aligned}
& {\left[\varrho^{\gamma-4} \exp \left(\frac{S}{c_{v} \varrho}\right)\right]^{2}\left\{\frac{\varrho^{2}}{c_{v}^{2}}\left[(\gamma-1) \varrho^{2}+\left((\gamma-1) \varrho-\frac{S}{c_{v}}\right)^{2}\right]-\left(\frac{\gamma-1}{c_{v}} \varrho^{2}-\frac{S}{c_{v}^{2}} \varrho\right)^{2}\right\}} \\
& =(\gamma-1) \frac{\varrho^{2 \gamma-4}}{c_{v}^{2}} \exp \left(\frac{2 S}{c_{v} \varrho}\right) .
\end{aligned}
$$

which completes the proof.

Consequently, we may define

$$
p(\varrho, S)=(\gamma-1) \varrho e(\varrho, S)=\left\{\begin{array}{l}
\varrho^{\gamma} \exp \left(\frac{S}{c_{v} \varrho}\right) \text { if } \varrho>0, S \in R, \\
0 \text { if } \varrho=0, S \leq 0, \\
\infty \text { if } \varrho=0, S>0,
\end{array}\right.
$$

which is a convex lower semi-continuous function on $[0, \infty) \times R$.

Similarly, we define the kinetic energy

$$
\frac{|\mathbf{m}|^{2}}{\varrho}=\left\{\begin{array}{l}
\frac{|\mathbf{m}|^{2}}{\varrho} \text { if } \varrho>0, \mathbf{m} \in R^{N} \\
0 \text { if } \mathbf{m}=0 \\
\infty \text { if } \varrho=0, \mathbf{m} \neq 0,
\end{array}\right.
$$

which is a convex lower semi-continuous function on $[0, \infty) \times R^{N}$. We conclude that the total energy

$$
\mathcal{E}=\mathcal{E}(\varrho, \mathbf{m}, S)=\frac{1}{2} \frac{|\mathbf{m}|^{2}}{\varrho}+\varrho e(\varrho, S)
$$

may be viewed as a convex lower semi-continuous function of $\varrho \geq 0, \mathbf{m} \in R^{N}$ and $S \in R$. 
3.2. Dissipative measure-valued solutions. Following [4] we introduce the concept of dissipative measure-valued solution to the Euler system (1.1)-(1.5). In addition, similarly to [2], we refine the definition of the measures describing the concentration defects. The reader may consult [4] for the physical background and mathematical objects like Young measures used in what follows.

We start by introducing the state space of "dummy variables":

$$
\mathcal{Q}=\left\{[\tilde{\varrho}, \tilde{\mathbf{m}}, \tilde{S}] \mid \tilde{\varrho} \geq 0, \tilde{\mathbf{m}} \in R^{N}, \tilde{S} \in R\right\}
$$

By $\mathcal{P}(\mathcal{Q})$ we denote the set of probability measures on $\mathcal{Q}$, whereas $\mathcal{M}^{+}(\bar{\Omega})$ and $\mathcal{M}^{+}\left(S^{N-1} \times \bar{\Omega}\right)$ denote the set of non-negative bounded Radon measures on $\bar{\Omega}$ and $S^{N-1} \times \bar{\Omega}$, respectively, where $S^{N-1} \subset R^{N}$ is the unit sphere.

A dissipative measure-valued solution of the Euler system (1.1)-(1.4) with the initial data

$\left[\varrho_{0}, \mathbf{m}_{0}, S_{0}\right]$ and the total energy $E_{0}$

consists of the following objects:

- a parametrized family of probability measures

$$
\mathcal{V}_{t, x}:(t, x) \in(0, \infty) \times \Omega \mapsto \mathcal{P}(\mathcal{Q}), \mathcal{V} \in L_{\mathrm{weak}-(*)}^{\infty}((0, T) \times \Omega ; \mathcal{P}(\mathcal{Q})) ;
$$

- kinetic and internal energy concentration defect measures

$$
\mathfrak{C}_{\mathrm{kin}}, \mathfrak{C}_{\mathrm{int}} \in L_{\mathrm{weak}-(*)}^{\infty}\left(0, \infty ; \mathcal{M}^{+}(\bar{\Omega})\right)
$$

- convective concentration defect measure

$$
\mathfrak{C}_{\mathrm{conv}} \in L_{\mathrm{weak}-(*)}^{\infty}\left(0, \infty ; \mathcal{M}^{+}\left(S^{N-1} \times \bar{\Omega}\right)\right), \frac{1}{2} \int_{S^{N-1}} \mathrm{~d} \mathfrak{C}_{\mathrm{conv}}=\mathfrak{C}_{\mathrm{kin}} .
$$

The Euler equations are satisfied in the following sense:

- The total energy is a constant of motion:

$$
\begin{aligned}
& \int_{\Omega}\left\langle\mathcal{V}_{t, x} ; \frac{1}{2} \frac{|\tilde{\mathbf{m}}|^{2}}{\tilde{\varrho}}+c_{v} \tilde{\varrho}^{\gamma} \exp \left(\frac{\tilde{S}}{c_{v} \tilde{\varrho}}\right)\right\rangle \mathrm{d} x \\
& +\int_{\bar{\Omega}}\left(\mathrm{d} \mathfrak{C}_{\text {kin }}(t)+\mathrm{d} \mathfrak{C}_{\text {int }}(t)\right)=E_{0} \text { for a.a. } t \geq 0 .
\end{aligned}
$$

- The conservation of mass (the continuity equation $\left.(1.1)_{1}\right)$ reads

$$
\int_{0}^{\infty} \int_{\Omega}\left[\left\langle\mathcal{V}_{t, x} ; \tilde{\varrho}\right\rangle \partial_{t} \varphi+\left\langle\mathcal{V}_{t, x} ; \tilde{\mathbf{m}}\right\rangle \cdot \nabla_{x} \varphi\right] \mathrm{d} x \mathrm{~d} t=-\int_{\Omega} \varrho_{0} \varphi(0) \mathrm{d} x
$$

for any $\varphi \in C_{c}^{1}([0, \infty) \times \bar{\Omega})$. 
- The balance of momentum (equation $(1.1)_{2}$ ) reads

$$
\begin{aligned}
& \int_{0}^{\infty} \int_{\Omega}\left[\left\langle\mathcal{V}_{t, x} ; \tilde{\mathbf{m}}\right\rangle \cdot \partial_{t} \boldsymbol{\varphi}+\left\langle\mathcal{V}_{t, x} ; \mathbb{I}_{\tilde{\varrho}>0} \frac{\tilde{\mathbf{m}} \otimes \tilde{\mathbf{m}}}{\tilde{\varrho}}\right\rangle: \nabla_{x} \boldsymbol{\varphi}\right] \mathrm{d} x \mathrm{~d} t \\
& +\int_{0}^{\infty} \int_{\Omega}\left[\left\langle\mathcal{V}_{t, x} ; p(\tilde{\varrho}, \tilde{S})\right\rangle \operatorname{div}_{x} \boldsymbol{\varphi}\right] \mathrm{d} x \mathrm{~d} t \\
& +\int_{0}^{\infty}\left[\int_{\bar{\Omega}} \int_{S^{N-1}}(\xi \otimes \xi): \nabla_{x} \boldsymbol{\varphi} \mathrm{d} \mathfrak{C}_{\text {conv }}(t)\right] \mathrm{d} t \\
& +(\gamma-1) \int_{0}^{\infty}\left[\int_{\bar{\Omega}} \operatorname{div}_{x} \boldsymbol{\varphi} \mathrm{d} \mathfrak{C}_{\text {int }}(t)\right] \mathrm{d} t=-\int_{\Omega} \mathbf{m}_{0} \boldsymbol{\varphi}(0) \mathrm{d} x
\end{aligned}
$$

for any $\boldsymbol{\varphi} \in C_{c}^{1}\left([0, \infty) \times \bar{\Omega} ; R^{N}\right),\left.\boldsymbol{\varphi} \cdot \mathbf{n}\right|_{\partial \Omega}=0$.

- The entropy balance (inequality (1.8)) is rewritten in the renormalized form:

$$
\begin{aligned}
& \int_{0}^{\infty} \int_{\Omega}\left[\left\langle\mathcal{V}_{t, x} ; \tilde{\varrho} Z\left(\frac{\tilde{S}}{\tilde{\varrho}}\right)\right\rangle \partial_{t} \varphi+\left\langle\mathcal{V}_{t, x} ; \mathbb{I}_{\tilde{\varrho}>0} Z\left(\frac{\tilde{S}}{\tilde{\varrho}}\right) \tilde{\mathbf{m}}\right\rangle \cdot \nabla_{x} \varphi\right] \mathrm{d} x \mathrm{~d} t \\
& \quad \leq-\int_{\Omega} \varrho_{0} Z\left(\frac{S_{0}}{\varrho_{0}}\right) \varphi(0) \mathrm{d} x
\end{aligned}
$$

for any $\varphi \in C_{c}^{1}([0, \infty) \times \bar{\Omega}), \varphi \geq 0$, and any $Z$,

$$
Z \in B C(R) \text { non-decreasing. }
$$

It follows from (3.2) and (3.3) that

$$
\begin{aligned}
t & \mapsto \int_{\Omega}\left\langle\mathcal{V}_{t, x} ; \tilde{\varrho}\right\rangle \varphi \mathrm{d} x, \varphi \in C^{1}(\bar{\Omega}) \\
t & \mapsto \int_{\Omega}\left\langle\mathcal{V}_{t, x} ; \tilde{\mathbf{m}}\right\rangle \varphi \mathrm{d} x, \varphi \in C^{1}\left(\bar{\Omega} ; R^{N}\right),\left.\boldsymbol{\varphi} \cdot \mathbf{n}\right|_{\partial \Omega}=0
\end{aligned}
$$

are continuous functions of time. Accordingly, equations (3.2) and (3.3) can be written as

$$
\int_{\tau_{1}}^{\tau_{2}} \int_{\Omega}\left[\left\langle\mathcal{V}_{t, x} ; \tilde{\varrho}\right\rangle \partial_{t} \varphi+\left\langle\mathcal{V}_{t, x} ; \tilde{\mathbf{m}}\right\rangle \cdot \nabla_{x} \varphi\right] \mathrm{d} x \mathrm{~d} t=\left[\int_{\Omega}\left\langle\mathcal{V}_{t, x} ; \tilde{\varrho}\right\rangle \varphi \mathrm{d} x\right]_{t=\tau_{1}}^{t=\tau_{2}},
$$

for any $0 \leq \tau_{1}<\tau_{2}<\infty$, and any $\varphi \in C_{c}^{1}([0, \infty) \times \bar{\Omega})$, where $\left\langle\mathcal{V}_{0, x} ; \tilde{\varrho}\right\rangle=\varrho_{0}(x)$;

$$
\begin{aligned}
& \int_{\tau_{1}}^{\tau_{2}} \int_{\Omega}\left[\left\langle\mathcal{V}_{t, x} ; \tilde{\mathbf{m}}\right\rangle \cdot \partial_{t} \boldsymbol{\varphi}+\left\langle\mathcal{V}_{t, x} ; \mathbb{I}_{\tilde{\varrho}>0} \frac{\tilde{\mathbf{m}} \otimes \tilde{\mathbf{m}}}{\tilde{\varrho}}\right\rangle: \nabla_{x} \boldsymbol{\varphi}\right] \mathrm{d} x \mathrm{~d} t \\
& +\int_{\tau_{1}}^{\tau_{2}} \int_{\Omega}\left\langle\mathcal{V}_{t, x} ; p(\tilde{\varrho}, \tilde{S})\right\rangle \operatorname{div}_{x} \boldsymbol{\varphi} \mathrm{d} x \mathrm{~d} t \\
& +\int_{\tau_{1}}^{\tau_{2}}\left[\int_{\bar{\Omega}} \int_{S^{N-1}}(\xi \otimes \xi): \nabla_{x} \boldsymbol{\varphi} \mathrm{d} \mathfrak{C}_{\text {conv }}(t)\right] \mathrm{d} t \\
& +(\gamma-1) \int_{\tau_{1}}^{\tau_{2}}\left[\int_{\bar{\Omega}} \operatorname{div}_{x} \boldsymbol{\varphi} \mathrm{d} \mathfrak{C}_{\mathrm{int}}(t)\right] \mathrm{d} t=\left[\int_{\Omega}\left\langle\mathcal{V}_{t, x} ; \tilde{\mathbf{m}}\right\rangle \boldsymbol{\varphi} \mathrm{d} x\right]_{t=\tau_{1}}^{t=\tau_{2}}, \\
& \left\langle\mathcal{V}_{0, x} ; \tilde{\mathbf{m}}\right\rangle=\mathbf{m}_{0}(x),
\end{aligned}
$$


for any $0 \leq \tau_{1}<\tau_{2}<\infty$, and any $\varphi \in C_{c}^{1}\left([0, \infty) \times \bar{\Omega} ; R^{N}\right),\left.\boldsymbol{\varphi} \cdot \mathbf{n}\right|_{\partial \Omega}=0$.

As shown in [4, Section 2.1.1], the renormalized entropy inequality (3.4) implies that

$$
\mathcal{V}_{t, x}\left\{(\varrho, S) \mid S \geq s_{0} \varrho>-\infty\right\}=1 \text { for a.a. }(t, x)
$$

as soon as

$$
S_{0} \geq \varrho_{0} s_{0} \text { a.a. in } \Omega .
$$

This is the minimum principle for the entropy $s=\frac{S}{\varrho} \geq s_{0}$. From now on, we fix $s_{0}$ and consider only solutions satisfying (3.7). This corresponds to having a new entropy $s-s_{0} \geq 0$. Then one can perform the limit passage $Z(s) \nearrow s$ in (3.4) obtaining the entropy balance

$$
\int_{0}^{\infty} \int_{\Omega}\left[\left\langle\mathcal{V}_{t, x} ; \tilde{S}\right\rangle \partial_{t} \varphi+\left\langle\mathcal{V}_{t, x} ; \mathbb{I}_{\tilde{\varrho}>0} \tilde{S} \frac{\tilde{\mathbf{m}}}{\tilde{\varrho}}\right\rangle \cdot \nabla_{x} \varphi\right] \mathrm{d} x \mathrm{~d} t \leq-\int_{\Omega} S_{0} \varphi(0) \mathrm{d} x,
$$

cf. [3]. In particular,

$$
t \mapsto \int_{\Omega}\left\langle\mathcal{V}_{t, x} ; \tilde{S}\right\rangle \varphi \mathrm{d} x=\chi_{\varphi}^{1}(t)+\chi_{\varphi}^{2}(t), \varphi \in C^{1}(\bar{\Omega}), \varphi \geq 0
$$

where $\chi_{\varphi}^{1}$ is continuous and $\chi_{\varphi}^{2}$ are non-decreasing. Thus (3.8) can be rewritten in the form

$$
\int_{\tau_{1}}^{\tau_{2}} \int_{\Omega}\left[\left\langle\mathcal{V}_{t, x} ; \tilde{S}\right\rangle \partial_{t} \varphi+\left\langle\mathcal{V}_{t, x} ; \mathbb{I}_{\tilde{\varrho}>0} \tilde{S} \frac{\tilde{\mathbf{m}}}{\tilde{\varrho}}\right\rangle \cdot \nabla_{x} \varphi\right] \mathrm{d} x \leq\left[\int_{\Omega}\left\langle\mathcal{V}_{t, x} ; \tilde{S}\right\rangle \varphi \mathrm{d} x\right]_{t=\tau_{1}-}^{t=\tau_{2}+}
$$

for any $0 \leq \tau_{1}<\tau_{2}<\infty$, and any $\varphi \in C^{1}([0, \infty) \times \bar{\Omega}), \varphi \geq 0$, where $\left\langle\mathcal{V}_{0-, x} ; \tilde{S}\right\rangle=$ $S_{0}(x)$.

Now we have all in hand to formulate the definition of a dissipative measure-valued solution.

Definition 3.2 (Dissipative measure-valued solution). A dissipative measure-valued solution of the Euler system (1.1)-(1.4) with the initial data

$$
\left[\varrho_{0}, \mathbf{m}_{0}, S_{0}\right] \text { and the energy } E_{0}
$$

is a parameterized family of probability measures

$$
\mathcal{V}_{t, x}:(t, x) \in(0, \infty) \times \Omega \mapsto \mathcal{P}(\mathcal{Q}), \mathcal{V} \in L_{\text {weak-(*) }}^{\infty}((0, T) \times \Omega ; \mathcal{P}(\mathcal{Q})),
$$

together with the energy concentration defect measures

$$
\mathfrak{C}_{\text {kin }}, \mathfrak{C}_{\text {int }} \in L_{\text {weak-(*) }}^{\infty}\left(0, \infty ; \mathcal{M}^{+}(\bar{\Omega})\right),
$$

and the convection concentration defect measure

$$
\mathfrak{C}_{\mathrm{conv}} \in L_{\text {weak }-(*)}^{\infty}\left(0, \infty ; \mathcal{M}^{+}\left(S^{N-1} \times \bar{\Omega}\right)\right), \frac{1}{2} \int_{S^{N-1}} \mathrm{~d} \mathfrak{C}_{\mathrm{conv}}=\mathfrak{C}_{\mathrm{kin}},
$$

satisfying the integral identities (3.1), (3.5), (3.6), and (3.9). 
Next, we list certain bounds that can be derived from hypothesis (3.7) and the energy equality (3.1). As the entropy is bounded below by $s_{0}$, we deduce from (3.1) that

$$
\int_{\Omega}\left\langle\mathcal{V}_{t, x} ; \tilde{\varrho}^{\gamma}\right\rangle \mathrm{d} x \lesssim E_{0} \text { for a.a. } t>0 .
$$

Similarly, as

$$
|\tilde{\mathbf{m}}|^{\frac{2 \gamma}{\gamma+1}}=|\tilde{\varrho}|^{\frac{\gamma}{\gamma+1}}\left|\frac{\tilde{\mathbf{m}}}{\sqrt{\tilde{\varrho}}}\right|^{\frac{2 \gamma}{\gamma+1}} \lesssim \tilde{\varrho}^{\gamma}+\frac{|\tilde{\mathbf{m}}|^{2}}{\tilde{\varrho}},
$$

we conclude

$$
\int_{\Omega}\left\langle\mathcal{V}_{t, x} ;|\tilde{\mathbf{m}}|^{\frac{2 \gamma}{\gamma+1}}\right\rangle \mathrm{d} x \lesssim E_{0} \text { for a.a. } t>0
$$

Finally, we derive some bounds on the total entropy $S$. Recall that $\tilde{S} \geq s_{0} \tilde{\varrho}$; whence

$$
|\tilde{S}|=-\tilde{S} \leq-s_{0} \tilde{\varrho} \text { whenever } \tilde{S} \leq 0
$$

If $\tilde{S}>0$, we compute

$$
\tilde{\varrho}^{\gamma} \exp \left(\frac{\tilde{S}}{c_{v} \tilde{\varrho}}\right)=c_{v}^{-\gamma} \frac{\exp \left(\frac{\tilde{S}}{c_{v} \tilde{\varrho}}\right)}{\left(\frac{\tilde{S}}{c_{v} \tilde{\varrho}}\right)^{\gamma}} \tilde{S}^{\gamma} \gtrsim \tilde{S}^{\gamma} .
$$

Consequently,

$$
\int_{\Omega}\left\langle\mathcal{V}_{t, x} ;|\tilde{S}|^{\gamma}\right\rangle \mathrm{d} x \lesssim E_{0} \text { for a.a. } t>0 .
$$

Next, we estimate the quantity $\tilde{S} / \sqrt{\tilde{\varrho}}$. If $\tilde{S} \leq 0$, we get, repeating the above argument,

$$
\left|\frac{\tilde{S}}{\sqrt{\tilde{\varrho}}}\right| \leq-s_{0} \sqrt{\tilde{\varrho}} \text { for } \tilde{S} \leq 0 .
$$

If $\tilde{S}>0$, we write

$$
\tilde{\varrho}^{\gamma} \exp \left(\frac{\tilde{S}}{c_{v} \tilde{\varrho}}\right)=\tilde{\varrho}^{\gamma} \exp \left(\frac{\tilde{S}}{\sqrt{\tilde{\varrho}}} \frac{1}{c_{v} \sqrt{\tilde{\varrho}}}\right)=c_{v}^{-2 \gamma} \frac{\exp \left(\frac{\tilde{S}}{\sqrt{\tilde{\varrho}}} \frac{1}{c_{v} \sqrt{\tilde{\varrho}}}\right)}{\left(\frac{\tilde{S}}{\sqrt{\tilde{\varrho}}} \frac{1}{c_{v} \sqrt{\tilde{\varrho}}}\right)^{2 \gamma}}\left(\frac{\tilde{S}}{\sqrt{\tilde{\varrho}}}\right)^{2 \gamma} \gtrsim\left(\frac{\tilde{S}}{\sqrt{\tilde{\varrho}}}\right)^{2 \gamma} \text {. }
$$

We may therefore infer that

$$
\int_{\Omega}\left\langle\mathcal{V}_{t, x} ;\left|\frac{\tilde{S}}{\sqrt{\tilde{\varrho}}}\right|^{2 \gamma}\right\rangle \mathrm{d} x \lesssim E_{0} \text { for a.a. } t>0 .
$$

Finally, we recall the weak-strong uniqueness principle proved in [4, Theorem 3.3]. 
Proposition 3.3. Let $\Omega \subset R^{N}, N=1,2,3$ be a bounded domain with smooth boundary. Suppose that the Euler system (1.1)-(1.5) admits a classical solution $[\varrho, \mathbf{m}, \mathcal{E}]$ in the class

$$
\varrho, \mathcal{E} \in C\left([0, T) ; W^{3,2}(\Omega)\right), \mathbf{m} \in C\left([0, T) ; W^{3,2}\left(\Omega ; R^{N}\right)\right)
$$

with the initial data

$$
\varrho_{0}>0, \mathbf{m}_{0}, \mathcal{E}_{0}=\frac{1}{2} \frac{\left|\mathbf{m}_{0}\right|^{2}}{\varrho_{0}}+c_{v} \varrho_{0} \vartheta_{0}, \vartheta_{0}>0 .
$$

Let $\mathcal{V}_{t, x}$ be a dissipative measure valued solution specified in Definition 3.2 starting from the data

$$
\varrho_{0}, \mathbf{m}_{0}, S_{0}=\varrho_{0} s\left(\varrho_{0}, \vartheta_{0}\right), E_{0}=\int_{\Omega} \mathcal{E}_{0} \mathrm{~d} x
$$

Then

$$
\left.\mathfrak{C}_{\text {kin }}\right|_{[0, T) \times \bar{\Omega}}=\left.\mathfrak{C}_{\text {int }}\right|_{[0, T) \times \bar{\Omega}}=0,\left.\mathfrak{C}_{\text {conv }}\right|_{[0, T) \times S^{N-1} \times \bar{\Omega}}=0,
$$

and

$$
\mathcal{V}_{t, x}=\delta_{[\varrho(t, x), \mathbf{m}(t, x), S(t, x)]}
$$

for a.a. $(t, x) \in[0, T) \times \Omega$.

Note that existence of a local-in-time classical solution in the class (3.14) was established by Schochet [18].

3.3. Dissipative solution. Having collected the necessary preliminary material, we are ready to introduce the central object of the present paper - the dissipative solutions to the Euler system.

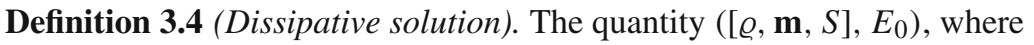

$$
\begin{gathered}
\varrho \in C_{\text {weak }, \mathrm{loc}}\left([0, \infty) ; L^{\gamma}(\Omega)\right), \mathbf{m} \in C_{\text {weak }, \mathrm{loc}}\left([0, \infty) ; L^{\frac{2 \gamma}{\gamma+1}}\left(\Omega ; R^{N}\right)\right), \\
S \in L^{\infty}\left(0, \infty ; L^{\gamma}(\Omega)\right) \cap \mathrm{BV}_{\text {weak }, \mathrm{loc}}\left([0, \infty) ; W^{-\ell, 2}(\Omega)\right), \ell>\frac{N}{2}+1,
\end{gathered}
$$

is a dissipative solution of the Euler system (1.1)-(1.4) with the initial data

$$
\left(\left[\varrho_{0}, \mathbf{m}_{0}, S_{0}\right], E_{0}\right) \in L^{\gamma}(\Omega) \times L^{\frac{2 \gamma}{\gamma+1}}\left(\Omega, R^{N}\right) \times L^{\gamma}(\Omega) \times[0, \infty)
$$

if there exists a dissipative measure-valued solution $\mathcal{V}_{t, x}$ as specified in Definition 3.2 such that

$$
\varrho(t, x)=\left\langle\mathcal{V}_{t, x} ; \tilde{\varrho}\right\rangle, \mathbf{m}(t, x)=\left\langle\mathcal{V}_{t, x} ; \tilde{\mathbf{m}}\right\rangle, S(t, x)=\left\langle\mathcal{V}_{t, x} ; \tilde{S}\right\rangle
$$


Remark 3.5. In accordance with the bounds (3.1), (3.10), (3.11), and (3.12), any dissipative solution belongs to the class

$$
\begin{gathered}
\varrho \in L^{\infty}\left([0, \infty) ; L^{\gamma}(\Omega)\right), \mathbf{m} \in L^{\infty}\left([0, \infty) ; L^{\frac{2 \gamma}{\gamma+1}}\left(\Omega ; R^{N}\right)\right), \\
S \in L^{\infty}\left([0, \infty) ; L^{\gamma}(\Omega)\right) ;
\end{gathered}
$$

whence to bounded balls in the afore mentioned spaces. These are compact metric (Polish) spaces with respect to the weak topology. In particular, condition (3.15) reduces to

$$
\begin{aligned}
t & \mapsto \int_{\Omega} \varrho \varphi \mathrm{d} x, t \mapsto \int_{\Omega} \mathbf{m} \cdot \varphi \mathrm{d} x \in C_{\mathrm{loc}}[0, \infty), \\
t & \mapsto \int_{\Omega} S \varphi \mathrm{d} x \in \mathrm{BV}_{\mathrm{loc}}[0, \infty) \forall \varphi \in C_{c}^{\infty}(\Omega), \varphi \in C_{c}^{\infty}\left(\Omega ; R^{N}\right) .
\end{aligned}
$$

Finally, we introduce a subclass of dissipative solutions that reflect the physical principle of maximal dissipation defined via the entropy production rate

$$
\sigma(\tau)=\int_{\Omega}\left(S(\tau)-S_{0}\right) \mathrm{d} x
$$

discussed in Sect. 1.2. Let $\left(\left[\varrho^{i}, \mathbf{m}^{i}, S^{i}\right], E_{0}\right), i=1,2$, be two dissipative solutions starting from the same initial data $\left(\left[\varrho_{0}, \mathbf{m}_{0}, S_{0}\right], E_{0}\right)$ with entropy production rates $\sigma^{1}$ and $\sigma^{2}$. Similarly to (1.11), we introduce the relation

$$
\begin{gathered}
\left(\left[\varrho^{1}, \mathbf{m}^{1}, S^{1}\right], E_{0}\right) \succ\left(\left[\varrho^{2}, \mathbf{m}^{2}, S^{2}\right], E_{0}\right) \\
\Leftrightarrow \sigma^{1}(\tau \pm) \geq \sigma^{2}(\tau \pm) \text { for any } \tau \in(0, \infty) .
\end{gathered}
$$

Definition 3.6 (Maximal dissipative solution). A dissipative solution ( $[\varrho, \mathbf{m}, S], E_{0}$ ) starting from the initial data $\left(\left[\varrho_{0}, \mathbf{m}_{0}, S_{0}\right], E_{0}\right)$ is a maximal dissipative solution if it is maximal with respect to the relation $\succ$. Specifically, if

$$
\left([\tilde{\varrho}, \tilde{\mathbf{m}}, \tilde{S}], E_{0}\right) \succ\left([\varrho, \mathbf{m}, S], E_{0}\right),
$$

where $\left([\tilde{\varrho}, \tilde{\mathbf{m}}, \tilde{S}], E_{0}\right)$ is another dissipative solution starting from $\left(\left[\varrho_{0}, \mathbf{m}_{0}, S_{0}\right], E_{0}\right)$, then

$$
\sigma=\tilde{\sigma} \text { in }[0, \infty) \text {. }
$$

Here $\sigma$ and $\tilde{\sigma}$ are the entropy production rates of $\left([\tilde{\varrho}, \tilde{\mathbf{m}}, \tilde{S}], E_{0}\right)$ and $([\varrho, \mathbf{m}, S], E)$ respectively.

3.4. Sequential stability. We start by introducing suitable topologies on the space of the initial data and the space of dissipative solutions. Fix $\ell>N / 2+1$ and consider the Hilbert space

$$
\mathcal{S}_{\text {Euler }}=W^{-\ell, 2}(\Omega) \times W^{-\ell, 2}\left(\Omega ; R^{N}\right) \times W^{-\ell, 2}(\Omega) \times R,
$$

together with its subset containing the initial data

$$
\begin{aligned}
\mathcal{D}_{\text {Euler }}= & \left\{\left(\left[\varrho_{0}, \mathbf{m}_{0}, S_{0}\right], E_{0}\right) \in L^{1}\left(\Omega ; R^{N+2}\right) \times R \mid\right. \\
& \left.\varrho_{0} \geq 0, S_{0} \geq s_{0} \varrho_{0}, \int_{\Omega}\left[\frac{1}{2} \frac{\left|\mathbf{m}_{0}\right|^{2}}{\varrho_{0}}+c_{v} \varrho_{0}^{\gamma} \exp \left(\frac{S_{0}}{c_{v} \varrho_{0}}\right)\right] \mathrm{d} x \leq E_{0}\right\}
\end{aligned}
$$


Note that $\mathcal{D}_{\text {Euler }}$ is a closed convex subset of $\mathcal{S}_{\text {Euler }}$. We also define the trajectory space

$$
\mathcal{T}_{\text {Euler }}=\mathrm{BV}_{\text {loc }}\left([0, \infty) ; \mathcal{S}_{\text {Euler }}\right)
$$

In the following we are going to show sequential stability (compactness) of the set of dissipative solutions. This will be subsequently used in the proof of existence of dissipative solutions (using a suitable approximation) as well as measurability of the mapping

$$
\begin{aligned}
\left(\left[\varrho_{0}, \mathbf{m}_{0}, S_{0}\right], E_{0}\right) \in \mathcal{D}_{\text {Euler }} & \mapsto \mathcal{U}\left(\left[\varrho_{0}, \mathbf{m}_{0}, S_{0}\right], E_{0}\right) \in 2^{\mathcal{T}_{\text {Euler }},} \\
& \mathcal{T}_{\text {Euler }}=L_{\text {loc }}^{1}\left([0, \infty) ; \mathcal{S}_{\text {Euler }}\right),
\end{aligned}
$$

where $\mathcal{U}\left(\left[\varrho_{0}, \mathbf{m}_{0}, S_{0}\right], E_{0}\right)$ denotes the solution set

$$
\mathcal{U}\left(\left[\varrho_{0}, \mathbf{m}_{0}, S_{0}\right], E_{0}\right)=\left\{\left[\varrho, \mathbf{m}, S, E_{0}\right] \in \mathcal{T}_{\text {Euler }} \mid\right.
$$

$\left([\varrho, \mathbf{m}, S], E_{0}\right)$ is a dissipative solution with initial data $\left.\left(\left[\varrho_{0}, \mathbf{m}_{0}, S_{0}\right], E_{0}\right)\right\}$

for the initial data $\left(\left[\varrho_{0}, \mathbf{m}_{0}, S_{0}\right], E_{0}\right) \in \mathcal{D}_{\text {Euler }}$. We have the following result.

Proposition 3.7. Suppose that $\left\{\left(\left[\varrho_{0, \varepsilon}, \mathbf{m}_{0, \varepsilon}, S_{0, \varepsilon}\right], E_{0, \varepsilon}\right)\right\}_{\varepsilon>0} \subset \mathcal{D}_{\text {Euler }}$ is a sequence of data giving rise to a family of dissipative solutions $\left\{\left(\left[\varrho_{\varepsilon}, \mathbf{m}_{\varepsilon}, S_{\varepsilon}\right], E_{0, \varepsilon}\right)\right\}_{\varepsilon>0}$, that is,

$$
\left(\left[\varrho_{\varepsilon}, \mathbf{m}_{\varepsilon}, S_{\varepsilon}\right], E_{0, \varepsilon}\right) \in \mathcal{U}\left(\left[\varrho_{0, \varepsilon}, \mathbf{m}_{0, \varepsilon}, S_{0, \varepsilon}\right], E_{0, \varepsilon}\right) .
$$

Moreover, we assume that there exists $\bar{E}>0$ such that $E_{0, \varepsilon} \leq \bar{E}$ for all $\varepsilon>0$.

Then, at least for suitable subsequences,

$$
\begin{aligned}
\varrho_{0, \varepsilon} & \rightarrow \varrho_{0} \text { weakly in } L^{\gamma}(\Omega), \mathbf{m}_{0, \varepsilon} \rightarrow \mathbf{m}_{0} \text { weakly in } L^{\frac{2 \gamma}{\gamma+1}}\left(\Omega ; R^{N}\right), \\
S_{0, \varepsilon} & \rightarrow S_{0} \text { weakly in } L^{\gamma}(\Omega), E_{0, \varepsilon} \rightarrow E_{0},
\end{aligned}
$$

and

$$
\begin{aligned}
\varrho_{\varepsilon} & \rightarrow \varrho \text { in } C_{\text {weak }, \mathrm{loc}}\left([0, \infty) ; L^{\gamma}(\Omega)\right), \\
\mathbf{m}_{\varepsilon} & \rightarrow \mathbf{m} \text { in } C_{\mathrm{weak}, \mathrm{loc}}\left([0, \infty) ; L^{\frac{2 \gamma}{\gamma+1}}\left(\Omega ; R^{N}\right)\right), \\
S_{\varepsilon} & \rightarrow S \text { in } L_{\mathrm{loc}}^{q}\left([0, \infty) ; W^{-\ell, 2}(\Omega)\right) \text { for any } q<\infty, \\
S_{\varepsilon} & \rightarrow S \text { weakly-(*) in } L^{\infty}\left(0, \infty ; L^{\gamma}(\Omega)\right),
\end{aligned}
$$

where

$$
\left([\varrho, \mathbf{m}, S], E_{0}\right) \in \mathcal{U}\left(\left[\varrho_{0}, \mathbf{m}_{0}, S_{0}\right], E_{0}\right) .
$$

Proof. We mainly follow the ideas from [2, Proposition 3.1], to which we refer for further details. Some modifications are needed to accommodate the additional variable $S$ (in the internal energy and the entropy balance).

First, we claim that the convergence in (3.17) follows immediately from the boundedness of the initial energy $E_{0, \varepsilon}$ and the uniform bounds (3.10)-(3.12). Indeed, using Jensen's inequality and convexity of the energy established in Lemma 3.1, we deduce that 


$$
\left[\frac{1}{2} \frac{\left|\mathbf{m}_{\varepsilon}\right|^{2}}{\varrho_{\varepsilon}}+p\left(\varrho_{\varepsilon}, S_{\varepsilon}\right)\right](t, x) \leq\left\langle\mathcal{V}_{t, x}^{\varepsilon} ; \frac{1}{2} \frac{|\tilde{\mathbf{m}}|^{2}}{\tilde{\varrho}}+p(\tilde{\varrho}, \tilde{S})\right\rangle
$$

for a.a. $(t, x) \in(0, \infty) \times \Omega$, where $\mathcal{V}_{t, x}^{\varepsilon}$ is the (Young) measure associated with the solution $\left(\left[\varrho_{\varepsilon}, \mathbf{m}_{\varepsilon}, S_{\varepsilon}\right], E_{0, \varepsilon}\right)$. Integrating over $\Omega$ we can see that the right hand side is bounded by $E_{0, \varepsilon} \leq \bar{E}$ using (3.1).

On the other, we can use (3.10)-(3.13) to obtain the estimates

$$
\begin{array}{r}
\sup _{t>0} \int_{\Omega}\left[\frac{\left|\mathbf{m}_{\varepsilon}\right|^{2}}{\varrho_{\varepsilon}}+\varrho_{\varepsilon}^{\gamma}+S_{\varepsilon}^{\gamma}\right] \mathrm{d} x \leq c(\bar{E}), \\
\sup _{t>0} \int_{\Omega}\left[\left|\mathbf{m}_{\varepsilon}\right|^{2 \gamma / \gamma+1}+\left|\frac{S_{\varepsilon}}{\sqrt{\varrho_{\varepsilon}}}\right|^{2 \gamma}\right] \mathrm{d} x \leq c(\bar{E}),
\end{array}
$$

uniformly in $\varepsilon$. Consequently, we deduce from equations (3.2), (3.3), and the energy balance (3.1), that

$$
\begin{gathered}
\varrho_{\varepsilon} \rightarrow \varrho \text { in } C_{\text {weak }, \text { loc }}\left([0, \infty) ; L^{\gamma}(\Omega)\right), \varrho \geq 0, \\
\mathbf{m}_{\varepsilon} \rightarrow \mathbf{m} \text { in } C_{\text {weak }, \text { loc }}\left([0, \infty) ; L^{\frac{2 \gamma}{\gamma+1}}\left(\Omega ; R^{N}\right)\right),
\end{gathered}
$$

where

$$
\varrho(0, \cdot)=\varrho_{0}, \mathbf{m}(0, \cdot)=\mathbf{m}_{0} .
$$

Similarly, we get from (3.19) that

$$
S_{\varepsilon} \rightarrow S \text { weakly-(*) in } L^{\infty}\left(0, \infty ; L^{\gamma}(\Omega)\right) .
$$

Moreover, we deduce from the entropy balance (3.9) and Helly's selection theorem that

$$
\int_{\Omega} S_{\varepsilon}(t, \cdot) \varphi \mathrm{d} x \rightarrow \int_{\Omega} S(t, \cdot) \varphi \mathrm{d} x \text { for any } t>0 \text { and any } \varphi \in W^{\ell, 2}(\Omega),
$$

modifying $S$ on the set of times of measure zero as the case may be (we split $\varphi=\varphi^{+}-\varphi^{-}$ and argue for both separately). Note that it is enough to show the former one for a dense subset of $W^{\ell, 2}(\Omega)$ which follows from a diagonal argument. Seeing that $L^{\gamma}(\Omega)$ endowed with the weak topology is compactly embedded into $W^{-\ell, 2}(\Omega)$ we obtain the desired conclusion

$$
S_{\varepsilon} \rightarrow S \text { in } L_{\text {loc }}^{q}\left(0, \infty ; W^{-\ell, 2}(\Omega)\right) \text { for any } 1 \leq q<\infty .
$$

Obviously,

$$
E_{0, \varepsilon} \rightarrow E_{0}
$$

passing to a subsequence as the case may be. Next, it is easy to perform the limit in the equation of continuity (3.2) to obtain (3.5).

Finally, as a consequence of the energy balance (3.1), the Young measures $\mathcal{V}_{t, x}^{\varepsilon}$ have uniformly bounded first moments; whence

$$
\mathcal{V}_{t, x}^{\varepsilon} \rightarrow \mathcal{V}_{t, x} \text { weakly-(*) in } L_{\mathrm{weak}-(*)}^{\infty}((0, \infty) \times \Omega ; \mathcal{P}(\mathcal{S}))
$$

at least for a subsequence. In addition, using estimates (3.11)-(3.13) we can pass to the limit in the entropy balance (3.9).

The limit in the total energy balance (3.1) and the momentum balance (3.3) involves the concentration measures and is more technical. However, the procedure is exactly the same as in [2, Section 3] therefore we omit the proof here. 
3.5. Existence of dissipative solutions. The sequential stability from the previous part combined with a suitable approximation implies the existence of a dissipative solution. The precise statement is the content of the following assertion.

Proposition 3.8. Let $\left(\left[\varrho_{0}, \mathbf{m}_{0}, S_{0}\right], E_{0}\right) \in \mathcal{D}_{\text {Euler }}$ be given. Then the Euler system (1.1)(1.4) admits a dissipative solution in the sense of Definition 3.4 with the initial data $\left(\left[\varrho_{0}, \mathbf{m}_{0}, S_{0}\right], E_{0}\right)$.

Proof. Given initial data $\left(\left[\varrho_{0}, \mathbf{m}_{0}, S_{0}\right], E_{0}\right) \in \mathcal{D}_{\text {Euler }}$ implies

$$
\varrho_{0} \in L^{\gamma}(\Omega), \mathbf{m}_{0} \in L^{\frac{2 \gamma}{\gamma+1}}\left(\Omega ; R^{N}\right), S_{0} \in L^{\gamma}(\Omega), \varrho_{0} \geq 0, S_{0} \geq s_{0} \varrho_{0},
$$

with the respective bounds in terms of $E_{0}$, recall the lower bounds for the energy obtained in (3.10)-(3.12). It is standard to construct smooth functions $\varrho_{0, \varepsilon}, \mathbf{m}_{0, \varepsilon}$ and $S_{0, \varepsilon}$, where $\varrho_{0, \varepsilon}$ is strictly positive and $\mathbf{m}_{0, \varepsilon}$ compactly supported in $\Omega, S_{0, \varepsilon} \geq \varrho_{0, \varepsilon} s_{0}$ such that

$$
\varrho_{0, \varepsilon} \rightarrow \varrho_{0} \text { in } L^{\gamma}(\Omega), \mathbf{m}_{0, \varepsilon} \rightarrow \mathbf{m}_{0} \text { in } L^{\frac{2 \gamma}{\gamma+1}}\left(\Omega ; R^{N}\right), S_{0, \varepsilon} \rightarrow S_{0} \text { in } L^{\gamma}(\Omega),
$$

and

$\int_{\Omega}\left[\frac{1}{2} \frac{\left|\mathbf{m}_{0, \varepsilon}\right|^{2}}{\varrho_{0, \varepsilon}}+c_{v} \varrho_{0, \varepsilon}^{\gamma} \exp \left(\frac{S_{0, \varepsilon}}{c_{v} \varrho_{0, \varepsilon}}\right)\right] \mathrm{d} x \rightarrow \int_{\Omega}\left[\frac{1}{2} \frac{\left|\mathbf{m}_{0}\right|^{2}}{\varrho_{0}}+c_{v} \varrho_{0}^{\gamma} \exp \left(\frac{S_{0}}{c_{v} \varrho_{0}}\right)\right] \mathrm{d} x$ as $\varepsilon \rightarrow 0$. Finally, we set $s_{0, \varepsilon}=S_{0, \varepsilon} / \varrho_{0, \varepsilon}$ and $\mathbf{u}_{0, \varepsilon}=\mathbf{m}_{0, \varepsilon} / \varrho_{0, \varepsilon}$.

Now, similarly to Kröner and Zajaczkowski [15], we consider the approximate system

$$
\begin{aligned}
\partial_{t} \varrho+\operatorname{div}_{x}(\varrho \mathbf{u}) & =0, \\
\partial_{t}(\varrho \mathbf{u})+\operatorname{div}_{x}(\varrho \mathbf{u} \otimes \mathbf{u})+\nabla_{x}\left(\varrho^{\gamma} \exp \left(\frac{s}{c_{v}}\right)\right) & =\varepsilon \mathcal{L} \mathbf{u}, \varepsilon>0, \\
\partial_{t} s+\mathbf{u} \cdot \nabla s & =0,
\end{aligned}
$$

with the initial data

$$
\varrho(0, \cdot)=\varrho_{0, \varepsilon}, \mathbf{u}(0, \cdot)=\mathbf{u}_{0, \varepsilon}, s(0, \cdot)=s_{0, \varepsilon} .
$$

Here, the symbol $\mathcal{L}$ denotes a suitable "viscosity" operator, with the associated set of boundary conditions to be imposed on the velocity field $\mathbf{u}$. In [15], the authors consider $\mathcal{L}=\Delta_{x}^{3}$ with the Dirichlet boundary conditions. This is not convenient here, as the resulting admissible test functions $\varphi$ in the momentum equation (3.3) would be compactly supported in $\Omega$. In order to preserve the weak-strong uniqueness principle (Proposition 3.3), however, we need a larger class of test functions satisfying merely the impermeability condition $\left.\boldsymbol{\varphi} \cdot \mathbf{n}\right|_{\partial \Omega}=0$. To this end, we use a different ansatz inspired by Kato and Lai [14].

Let $W_{n}^{3,2}\left(\Omega ; R^{N}\right)$ be the Hilbert space,

$$
W_{n}^{3,2}\left(\Omega ; R^{N}\right)=\left\{\mathbf{u} \in W^{3,2}\left(\Omega ; R^{N}\right)|\mathbf{u} \cdot \mathbf{n}|_{\partial \Omega}=0\right\} .
$$

We suppose that $\Omega \subset R^{N}, N=1,2,3$ is a bounded regular domain so that $W_{n}^{3,2}\left(\Omega ; R^{N}\right)$ is compactly embedded in $C^{1}\left(\bar{\Omega} ; R^{N}\right)$. Moreover, $W_{n}^{3,2}$ is separable as being a closed 
subset of the separable Hilbert space $W^{3,2}$. Let $((;))$ be the scalar product on $W_{n}^{3,2}$, that is,

$$
((\mathbf{v} ; \mathbf{w}))=\sum_{|\alpha|=3} \int_{\Omega} \partial_{x}^{\alpha} \mathbf{v} \cdot \partial_{x}^{\alpha} \mathbf{w} \mathrm{d} x+\int_{\Omega} \mathbf{v} \cdot \mathbf{w} \mathrm{d} x, \mathbf{v}, \mathbf{w} \in W_{n}^{3,2}\left(\Omega ; R^{N}\right) .
$$

Similarly to [14], we consider $\mathcal{L}$ to be the self-adjoint operator on $W_{n}^{3,2}$ associated to the bilinear form $((;))$, namely

$$
\mathcal{L} \mathbf{u}=\sum_{|\alpha|=3}\left(-\partial_{x}^{\alpha}\right) \partial_{x}^{\alpha} \mathbf{u}+\mathbf{u} \text { with the homogeneous Neumann boundary conditions. }
$$

The associated variational formulation of the momentum equation in (3.22) then reads

$$
\begin{aligned}
{\left[\int_{\Omega} \varrho \mathbf{u} \cdot \boldsymbol{\varphi} \mathrm{d} x\right]_{t=0}^{t=\tau} } & =\int_{\Omega}\left[\varrho \mathbf{u} \otimes \mathbf{u}: \nabla_{x} \boldsymbol{\varphi}+\varrho^{\gamma} \exp \left(\frac{s}{c_{v}}\right) \operatorname{div}_{x} \boldsymbol{\varphi}\right] \mathrm{d} x \\
& -\varepsilon((\mathbf{u} ; \boldsymbol{\varphi}))
\end{aligned}
$$

for any $\tau>0$, and any $\varphi \in W_{n}^{3,2}\left(\Omega ; R^{N}\right)$.

As $W_{n}^{3,2}$ is a separable Hilbert space, the existence proof used in [15, Theorem 4.1] applies without essential modifications (see also Hoff [13] for how to handle the transport equations for $\varrho$ and $s)$. Given $\varepsilon>0$, there exists a trio $\left(\varrho_{\varepsilon}, \mathbf{u}_{\varepsilon}, s_{\varepsilon}\right)$ which is a classical solution to (3.22) in the following sense:

- The balance of mass $(3.22)_{1}$ and the entropy balance $(3.22)_{3}$ hold pointwise in $(0, \infty) \times \Omega$.

- The balance of momentum (3.22) 2 holds in the sense of (3.24).

- The solution possesses enough regularity to rigorously justify the standard energy estimate

$$
\frac{\mathrm{d}}{\mathrm{d} t} \int_{\Omega}\left[\frac{1}{2} \varrho_{\varepsilon}\left|\mathbf{u}_{\varepsilon}\right|^{2}+c_{v} \varrho_{\varepsilon}^{\gamma} \exp \left(\frac{s_{\varepsilon}}{c_{v}}\right)\right] \mathrm{d} x+\varepsilon\left(\left(\mathbf{u}_{\varepsilon} ; \mathbf{u}_{\varepsilon}\right)\right)^{2}=0 .
$$

Finally, we set $S_{\varepsilon}=\varrho_{\varepsilon} s_{\varepsilon}$ and $\mathbf{m}_{\varepsilon}=\varrho_{\varepsilon} \mathbf{u}_{\varepsilon}$. Using the arguments of the preceding section, it is easy to perform the limit $\varepsilon \rightarrow 0$ in the sequence of approximate solutions

$$
\left\{\varrho_{\varepsilon}, \mathbf{m}_{\varepsilon}=\varrho_{\varepsilon} \mathbf{u}_{\varepsilon}, S_{\varepsilon}=\varrho_{\varepsilon} s_{\varepsilon}, E_{\varepsilon}=\int_{\Omega}\left[\frac{1}{2} \varrho_{\varepsilon}\left|\mathbf{u}_{\varepsilon}\right|^{2}+c_{v} \varrho_{\varepsilon}^{\gamma} \exp \left(\frac{S_{\varepsilon}}{c_{v} \varrho_{\varepsilon}}\right)\right] \mathrm{d} x\right\}_{\varepsilon>0}
$$

to obtain the desired dissipative solution, with the exception of the energy equality (3.1) that now reads

$$
\begin{aligned}
& \int_{\Omega}\left\langle\mathcal{V}_{t, x} ; \frac{1}{2} \frac{|\tilde{\mathbf{m}}|^{2}}{\tilde{\varrho}}+c_{v} \tilde{\varrho}^{\gamma} \exp \left(\frac{\tilde{S}}{c_{v} \tilde{\varrho}}\right)\right\rangle \mathrm{d} x \\
& +\int_{\bar{\Omega}}\left(\mathrm{d} \mathfrak{C}_{\text {kin }}(t)+\mathrm{d} \mathfrak{C}_{\text {int }}(t)\right) \leq E_{0} \text { for a.a. } t \geq 0 .
\end{aligned}
$$

Note that, on account of (3.25), it is easy to see that viscous term in the momentum equation vanishes as $\varepsilon \rightarrow 0$. In contrast with (3.26), the entropy balance (3.9) holds as equality. To convert (3.26) to equality, it is enough to augment $\mathfrak{C}_{\text {int }}(t)$ by $h(t) \mathrm{d} x$ with a 
suitable spatially homogeneous $h \geq 0$. Note that the momentum equation (3.3) remains valid as $\mathfrak{C}_{\text {int }}(t)$ acts on $\operatorname{div}_{x} \boldsymbol{\varphi}$, where $\left.\boldsymbol{\varphi} \cdot \mathbf{n}\right|_{\partial \Omega}=0$; whence

$$
\int_{\Omega} h(t) \operatorname{div}_{x} \varphi \mathrm{d} x=0 .
$$

\section{Semiflow Selection}

The goal of this section is to show that there is a semiflow selection to the Euler system (1.1)-(1.4). We recall that the precise definition of a semiflow - in the abstract framework - is given in Definition 2.1 (recall Subsection 3.4 for the definitions of $\mathcal{D}_{\text {Euler }}$ and $\mathcal{T}_{\text {Euler }}$ ). The following is the main result of the present paper.

Theorem 4.1 (Semiflow solution) The Euler system (1.1)-(1.4) admits a semiflow solution in the class of dissipative solutions in the sense of Definition 3.4. More specifically, for any initial data

$$
\mathbb{U}_{0}=\left(\left[\varrho_{0}, \mathbf{m}_{0}, S_{0}\right], E_{0}\right) \in \mathcal{D}_{\text {Euler }}
$$

there exists a dissipative solution

$$
\mathbb{U}=\mathbb{U}\left[t, \mathbb{U}_{0}\right]=\left([\varrho(t, \cdot), \mathbf{m}(t, \cdot), S(t-, \cdot)], E_{0}\right) \in \mathcal{T}_{\text {Euler }}
$$

enjoying the following properties:

- for each $\mathbb{U}_{0} \in \mathcal{D}_{\text {Euler }}$ the solution $\mathbb{U}\left[\cdot, \mathbb{U}_{0}\right]$ is maximal in the sense of Definition 3.6;

- $\mathcal{D}_{\text {Euler }}$ is an invariant set, meaning

$$
\mathbb{U}\left[t, \mathbb{U}_{0}\right] \in \mathcal{D}_{\text {Euler }}
$$

for any $t \geq 0$;

- the mapping

$$
\mathbb{U}_{0} \in \mathcal{S}_{\text {Euler }} \mapsto \mathbb{U}\left[\cdot, \mathbb{U}_{0}\right] \in L_{\text {loc }}^{1}\left(0, \infty ; \mathcal{S}_{\text {Euler }}\right)
$$

is Borel measurable;

- the mapping

$$
\mathbb{U}:[0, \infty) \times \mathcal{D}_{\text {Euler }} \mapsto \mathcal{D}_{\text {Euler }}
$$

is a semiflow, specifically,

$$
\mathbb{U}\left[t_{1}+t_{2} ; \mathbb{U}_{0}\right]=\mathbb{U}\left[t_{2} ;\left[\mathbb{U}\left[t_{1} ; \mathbb{U}_{0}\right]\right]\right.
$$

for any $t_{1}, t_{2} \geq 0$, and any $\mathbb{U}_{0} \in \mathcal{D}_{\text {Euler. }}$.

The claim of Theorem 4.1 will follow from Proposition 2.3 as soon as we verify [A1]-[A4] from Definition 2.2 for the solution set $\mathcal{U}\left(\left[\varrho_{0}, \mathbf{m}_{0}, S_{0}\right], E_{0}\right)$ with $\left(\left[\varrho_{0}, \mathbf{m}_{0}, S_{0}\right], E_{0}\right) \in \mathcal{D}_{\text {Euler. }}$ This will be done in the following lemmas.

Lemma 4.2. For each $\left(\left[\varrho_{0}, \mathbf{m}_{0}, S_{0}\right], E_{0}\right) \in \mathcal{D}_{\text {Euler }}$ the set $\mathcal{U}\left[\varrho_{0}, \mathbf{m}_{0}, S_{0}, E_{0}\right]$ is a nonempty bounded subset of $\mathrm{BV}_{\mathrm{loc}}\left([0, \infty) ; \mathcal{S}_{\text {Euler }}\right)$ and it is compact in the topology of $L_{\text {loc }}^{1}\left([0, \infty) ; \mathcal{S}_{\text {Euler }}\right)$. 
Proof. The claim follows from Propositions 3.7 and 3.8.

Lemma 4.3. Let $\left([\varrho, \mathbf{m}, S], E_{0}\right)$ be a dissipative solution to the Euler system in the sense of Definition 3.4. Then $\mathfrak{S}_{T} \circ\left([\varrho, \mathbf{m}, S], E_{0}\right)$ is a dissipative solution corresponding to the data $\left([\varrho, \mathbf{m}, S](T-), E_{0}\right)$.

Proof. We recall that the operation of time shift is given by

$$
\mathfrak{S}_{T} \circ\left([\varrho, \mathbf{m}, S], E_{0}\right)(t)=\left([\varrho, \mathbf{m}, S](T+t), E_{0}\right), T>0, t \geq 0 .
$$

Since a shift of a test function in (3.1)-(3.4) produces a test function in the same class, the claim easily follows.

Lemma 4.4. Let $\left(\left[\varrho^{1}, \mathbf{m}^{1}, S^{1}\right], E_{0}\right)$ be a dissipative solution of the Euler system, $T>0$ and let $\left(\left[\varrho^{2}, \mathbf{m}^{2}, S^{2}\right], E_{0}\right)$ be another dissipative solution with the initial data $\left(\left[\varrho^{1}, \mathbf{m}^{1}, S^{1}\right](T-), E_{0}\right)$.

Then

$$
\left(\left[\varrho^{1}, \mathbf{m}^{1}, S^{1}\right], E_{0}\right) \cup_{T}\left(\left[\varrho^{2}, \mathbf{m}^{2}, S^{2}\right], E_{0}\right)
$$

is a dissipative solution of the Euler system.

Proof. The concatenation property for (3.1)-(3.3) is obvious. As far as (3.4) is concerned we observe the following. The function

$$
[\varrho, S] \mapsto \varrho Z\left(\frac{S}{\varrho}\right)
$$

is concave such that

$$
\int_{\Omega} \varrho Z\left(\frac{S}{\varrho}\right)(T-) \varphi \mathrm{d} x \geq \limsup _{t \rightarrow T-} \int_{\Omega}\left\langle\mathcal{V}_{t, x} ; \varrho Z\left(\frac{S}{\varrho}\right)\right\rangle \varphi \mathrm{d} x .
$$

So, (3.4) has the concatenation property as well.

\section{Lemma 4.5. The mapping}

$$
\mathcal{U}:\left(\left[\varrho_{0}, \mathbf{m}_{0}, S_{0}\right], E_{0}\right) \in \mathcal{D}_{\text {Euler }} \mapsto \mathcal{U}\left(\left[\varrho_{0}, \mathbf{m}_{0}, S_{0}\right], E_{0}\right) \in 2^{\mathcal{T}_{\text {Euler }}}
$$

is Borel measurable; where $2^{\mathcal{T}_{\text {Euler }}}$ is endowed with the Hausdorff topology defined on compact subsets of the metric space $L_{\text {loc }}^{1}\left([0, \infty) ; \mathcal{S}_{\text {Euler }}\right)$.

Proof. As a consequence of the sequential stability from Propositions 3.7 the claim follows from [20, Thm. 12.1.8].

Proof of Theorem 4.1. In view of Proposition 2.3, the first claim of Theorem 4.1- the existence of the semiflow $\mathbb{U}$-follows now from Lemmas 4.2-4.5. Let us finally explain why $\mathbb{U}\left(\left[\varrho_{0}, \mathbf{m}_{0}, S_{0}\right], E_{0}\right)$ can be selected to be maximal in the sense of Definition 3.6. As stated in Proposition 2.3 the selection can be chosen to satisfy

$$
\begin{aligned}
& \int_{0}^{\infty} \exp (-t) \beta\left(\mathbb{U}\left(\left[\varrho_{0}, \mathbf{m}_{0}, S_{0}\right], E_{0}\right)(t)\right) \mathrm{d} t \\
& \quad \leq \int_{0}^{\infty} \exp (-t) \beta\left(\left([\tilde{\varrho}, \tilde{\mathbf{m}}, \tilde{S}], E_{0}\right)(t)\right) \mathrm{d} t .
\end{aligned}
$$


for any $\left([\tilde{\varrho}, \tilde{\mathbf{m}}, \tilde{S}], E_{0}\right) \in \mathcal{U}\left(\left[\varrho_{0}, \mathbf{m}_{0}, S_{0}\right], E_{0}\right)$. Here $\beta: \mathcal{S}_{\text {Euler }} \rightarrow R$ is a bounded and continuous function. We suppose that

$$
\beta(\varrho, \mathbf{m}, S, E)=\alpha\left(\int_{\Omega} S \mathrm{~d} x\right),
$$

where $\alpha: R \rightarrow R$ is smooth, bounded and strictly decreasing. We proceed by contradiction. Let $\left([\tilde{\varrho}, \tilde{\mathbf{m}}, \tilde{S}], E_{0}\right) \in \mathcal{U}\left(\left[\varrho_{0}, \mathbf{m}_{0}, S_{0}\right], E_{0}\right)$ be such that $\left([\tilde{\varrho}, \tilde{\mathbf{m}}, \tilde{S}], E_{0}\right) \succ$ $\left([\varrho, \mathbf{m}, S], E_{0}\right)$, that is, $\int_{\Omega} \tilde{S} \mathrm{~d} x \geq \int_{\Omega} S \mathrm{~d} x$ in $(0, \infty)$, where we denote $\left([\varrho, \mathbf{m}, S], E_{0}\right)=$ $\mathbb{U}\left(\left[\varrho_{0}, \mathbf{m}_{0}, S_{0}\right], E_{0}\right)$. Then we get

$$
\begin{gathered}
\alpha\left(\int_{\Omega} \tilde{S} \mathrm{~d} x\right) \leq \alpha\left(\int_{\Omega} S \mathrm{~d} x\right), \\
\int_{0}^{\infty} \exp (-t)\left[\alpha\left(\int_{\Omega} S \mathrm{~d} x\right)-\alpha\left(\int_{\Omega} \tilde{S} \mathrm{~d} x\right)\right] \mathrm{d} t \leq 0
\end{gathered}
$$

whence $\int_{\Omega} S \mathrm{~d} x=\int_{\Omega} \tilde{S} \mathrm{~d} x$ a.a. in $(0, \infty)$ since $\alpha$ is strictly decreasing. Hence $\mathbb{U}\left(\left[\varrho_{0}, \mathbf{m}_{0}, S_{0}\right], E_{0}\right)$ is maximal with respect to $\succ$. The proof of Theorem 4.1 is hereby complete.

\section{Concluding Discussion}

We have shown the existence of a semiflow solution to the complete Euler system in the class of dissipative solutions. The semiflow solution has been selected among other solutions starting from the same initial data. The major issue is, of course, uniqueness of such a selection. In accordance with Proposition 3.3 the selected solution is unique and coincides with the strong solution emanating from the same initial state as long as the latter exists. There is a special class of strong solutions - the equilibrium states. As we shall see below, they enjoy certain stability properties thanks to the fact that the selected solutions produce the maximal amount of entropy.

5.1. Stability of equilibrium states. The equilibrium states are stationary solutions to the Euler system (1.1)-(1.4). Specifically, the momentum vanishes identically $\mathbf{m} \equiv 0$, while the density $\varrho=\bar{\varrho}>0$ and the entropy $S=\bar{S}$ are constant. Note that $\bar{\varrho}$ is uniquely determined by the total mass

$$
M=\bar{\varrho}|\Omega|=\int_{\Omega} \varrho(t, x) \mathrm{d} x=\int_{\Omega} \varrho_{0}(x) \mathrm{d} x \text { for any } t>0 .
$$

Given $\bar{\varrho}$ and the total energy $E_{0}$, the (constant) equilibrium entropy $\bar{S}$ is the unique maximizer of the total entropy among all states with given mass and energy:

$$
\begin{array}{r}
\int_{\Omega} \bar{S} \mathrm{~d} x=\sup \left\{\int_{\Omega} S \mathrm{~d} x \mid \varrho, S \in L^{1}(\Omega), \varrho \geq 0,\right. \\
\left.\int_{\Omega} c_{v} \varrho^{\gamma} \exp \left(\frac{S}{c_{v} \varrho}\right) \mathrm{d} x=E_{0}, \int_{\Omega} \varrho \mathrm{d} x=M\right\} .
\end{array}
$$

Indeed, suppose that $\bar{\varrho}, \bar{S}$ are constant, and

$$
\begin{gathered}
\int_{\Omega} \bar{\varrho} \mathrm{d} x=\int_{\Omega} \varrho \mathrm{d} x=M, \int_{\Omega} \bar{S} \mathrm{~d} x \leq \int_{\Omega} S \mathrm{~d} x, \\
\int_{\Omega} \bar{\varrho} e(\bar{\varrho}, \bar{S}) \mathrm{d} x=\int_{\Omega} \varrho e(\varrho, S) \mathrm{d} x=E_{0} .
\end{gathered}
$$


Normalizing the above relation by a factor $\frac{1}{|\Omega|}$ we may suppose $|\Omega|=1$. Using convexity of the function $\Phi(\varrho, S) \equiv \varrho e(\varrho, S)$ (cf. Lemma 3.1) and Jensen's inequality, we get

$$
\begin{aligned}
E_{0} & =\int_{\Omega} \Phi(\bar{\varrho}, \bar{S}) \mathrm{d} x=\Phi\left(\int_{\Omega} \bar{\varrho} \mathrm{d} x, \int_{\Omega} \bar{S} \mathrm{~d} x\right) \\
& \leq \Phi\left(\int_{\Omega} \varrho \mathrm{d} x, \int_{\Omega} S \mathrm{~d} x\right) \leq \int_{\Omega} \Phi(\varrho, S) \mathrm{d} x=E_{0} .
\end{aligned}
$$

As $\Phi$ is strictly increasing in $S$ and strictly convex in $(\varrho, S)$, we get successively $\int_{\Omega} S \mathrm{~d} x=\int_{\Omega} \bar{S} \mathrm{~d} x$, and $\varrho=\bar{\varrho}, S=\bar{S}$.

We claim that a maximal solution can only see the equilibria with the energy $E_{0}$.

Proposition 5.1 (Stability of equilibria). Let $\left([\varrho, \mathbf{m}, S], E_{0}\right)$ be a dissipative solution to the Euler system emanating from the initial data

$$
\varrho_{0}=\bar{\varrho}-\text { a positive constant, } \mathbf{m}_{0}=0, S_{0}-\text { a constant } .
$$

Suppose that $\left([\varrho, \mathbf{m}, S], E_{0}\right)$ is maximal in the sense of Definition 3.6.

Then

$$
\varrho=\bar{\varrho}, \mathbf{m}=0, S=\bar{S}, \text { where } \int_{\Omega} c_{v} \bar{\varrho}^{\gamma} \exp \left(\frac{S}{c_{v} \bar{\varrho}}\right) \mathrm{d} x=\bar{S} .
$$

Proof. As the equilibrium solution maximizes the total entropy, we have $\bar{S} \geq S_{0}$; whence (5.1) is a dissipative solution. By the same token, it is a maximal solution and any other solution "larger" in the sense of $\succ$ has the same entropy, meaning it concides with the solution (5.1).

5.2. Dafermos' admissibility criteria. Finally, we discuss some implications of Dafermos' admissibility criteria introduced in Sect. 1.1. As we have seen in the proof of Theorem 4.1, maximimality of the entropy production by dissipative solutions has been enforced by maximizing the integral

$$
\int_{0}^{\infty} \exp (-t) \int_{\Omega} S(t, x) \mathrm{d} x \mathrm{~d} t
$$

modulo the cut-off function $\alpha$. Actually the same result could have been achieved by maximizing

$$
\int_{0}^{\infty} \exp (-\lambda t) \int_{\Omega} S(t, x) \mathrm{d} x \mathrm{~d} t, \lambda>0 .
$$

Thus, a natural question arises, namely, what would be the resulting solution if we maximized successively the entropy for a family $\lambda_{n} \rightarrow \infty$ ? Lemma 5.2 below implies the following: if there is a solution such that it has maximal Laplace transform of the total entropy evaluated at every $\lambda_{n}$ from a sequence $\lambda_{n} \rightarrow \infty$, then it satisfies the entropy production criterium by Dafermos in the form (1.9), that is, it is maximal with respect to the partial ordering $\succ_{\mathcal{D}}$.

With this motivation in mind, we employ the following notation for $F, G \in$ $L^{\infty}(0, \infty) \cap \mathrm{BV}_{\text {loc }}([0, \infty))$ : We say that $F \succ_{\mathcal{D}} G$ provided there exists $\delta>0$ such that $F(t+) \geqslant G(t+)$ for all $t \in(0, \delta)$; and we say that $F \succ_{\mathcal{F}} G$ provided there exists a sequence $\left(\tau_{n}\right)_{n}, \tau_{n}>0, \tau_{n} \rightarrow 0$ such that $F\left(\tau_{n}+\right) \geqslant G\left(\tau_{n}+\right)$. 
Lemma 5.2. Let $\mathcal{G} \subset L^{\infty}(0, \infty) \cap \mathrm{BV}_{\text {loc }}([0, \infty))$ and assume that there exists $F \in \mathcal{G}$ such that for some sequence $\lambda_{n} \rightarrow \infty$ it holds

$$
\lambda_{n} \int_{0}^{\infty} \exp \left(-\lambda_{n} t\right) F(t) \mathrm{d} t \geqslant \lambda_{n} \int_{0}^{\infty} \exp \left(-\lambda_{n} t\right) G(t) \mathrm{d} t \quad \text { for all } G \in \mathcal{G} \text { and } n \in \mathbb{N} \text {. }
$$

Then for every $G \in \mathcal{G} \backslash\{F\}$ one of the following cases holds: either $F \succ_{\mathcal{D}} G$ or $F \sim_{\mathcal{F}} G$, meaning, in the latter case it holds simultaneously $F \succ_{\mathcal{F}} G$ and $G \succ_{\mathcal{F}} F$. Accordingly, $F$ is maximal with respect to $\succ_{\mathcal{D}}$.

Proof. Fix an arbitrary $G \in \mathcal{G} \backslash\{F\}$ and assume that the first claim $F \succ_{\mathcal{D}} G$ is not valid. That is, for every $\delta>0$ there exists $t \in(0, \delta)$ such that $F(t+)<G(t+)$ This directly implies $G \succ_{\mathcal{F}} F$. So it remains to show the converse statement, i.e. $F \succ_{\mathcal{F}} G$.

Case 1: If there is a $\delta>0$ such that $F(t+)<G(t+)$ for all $t \in(0, \delta)$ then we write

$$
\begin{aligned}
& \lambda_{n} \int_{0}^{\infty} \exp \left(-\lambda_{n} t\right)[G(t)-F(t)] \mathrm{d} t \\
& \quad=\lambda_{n} \int_{0}^{\delta} \exp \left(-\lambda_{n} t\right)[G(t)-F(t)] \mathrm{d} t+\lambda_{n} \int_{\delta}^{\infty} \exp \left(-\lambda_{n} t\right)[G(t)-F(t)] \mathrm{d} t \\
& \quad \geqslant \lambda_{n} \exp \left(-\lambda_{n} \delta\right) \int_{0}^{\delta}[G(t)-F(t)] \mathrm{d} t-\left(\|F\|_{L^{\infty}}+\|G\|_{L^{\infty}}\right) \exp \left(-\lambda_{n} \delta\right),
\end{aligned}
$$

where the right hand side is strictly positive provided $n$ was chosen sufficiently large. This is a contradiction with the maximality of the corresponding Laplace transforms of $F$.

Case 2: If that is not the case, then the functions $F, G$ oscillate around each other in the sense that for every $\delta>0$ there exists $t \in(0, \delta)$ such that $F(t+) \geqslant G(t+)$. Hence $F>\mathcal{F} G$ and the second claim, namely, $F \sim_{\mathcal{F}} G$, is valid.

Finally, as already observed in Sect. 1.1, maximality with respect to $\succ_{\mathcal{F}}$ implies maximality with respect to $\succ_{\mathcal{D}}$. Thus, $F$ is maximal with respect to $\succ_{\mathcal{D}}$ and the proof is complete.

In other words, if for some initial data the Euler system (1.1)-(1.4) possesses a solution whose total entropy has maximal Laplace transforms evaluated at a sequence $\lambda_{n} \rightarrow \infty$, then the Dafermos' criterium is satisfied. Note that since we have a semigroup it is enough to test the criterium at the time $t=0$.

We now argue that such a solution can be always chosen by our selection process considering a suitable order of minimizers in the procedure described in [2, Section 5.1]. The latter one considers bounded continuous functionals $\beta: \mathcal{S} \rightarrow R$ on the phase space. The final selection $\mathbb{U}$ maximizes (or minimizes) $\beta(\mathbb{U})$ pointwise in time by selecting the maximizer (or minimizer) of the functionals

$$
I_{n}[\mathbb{V}]=\int_{0}^{\infty} \exp \left(-\lambda_{n} t\right) \beta(\mathbb{V}) \mathrm{d} t, \mathbb{V} \in \mathrm{BV}_{\text {loc }}([0, \infty) ; \mathcal{S}),
$$

from the solution set. In [2, Section 5.1], the sequence $\left(\lambda_{n}\right)_{n}$ is chosen to be dense in $(0, \infty)$. However, the density of $\left(\lambda_{n}\right)_{n}$ is only needed to apply Lerch's theorem implying the uniqueness of the Laplace transform. In fact, it is sufficient that the family of functionals $I_{n}$ in (5.3) separates points, which can be achieved by choosing a suitable increasing sequence $\lambda_{n} \rightarrow \infty$. This follows from the following generalization of the classical Lerch's theorem. Consequently, it is enough that the total entropy of our selection maximizes (5.2) for $\lambda=\lambda_{n}, n \in \mathbb{N}$. 
Lemma 5.3. Let $\lambda>0$ and $\left(\zeta_{n}\right)_{n} \subset(0, \infty)$ be a sequence with the following property: for all $n, m \in \mathbb{N}$ there exists $k \in \mathbb{N}$ such that $\zeta_{n}+\zeta_{m}=\zeta_{k}$. Then the set of functionals

$$
L^{\infty}(0, \infty) \rightarrow \mathbb{R}, \quad F \mapsto \int_{0}^{\infty} \exp \left(-\left(\lambda+\zeta_{n}\right) t\right) F(t) \mathrm{d} t, \quad n \in \mathbb{N},
$$

separates points.

Proof. Due to the condition on the sequence $\left(\zeta_{n}\right)_{n}$, the set of finite linear combinations of functions $\left(e^{-\zeta_{n} t}\right)_{n}$ forms a subalgebra of $C_{0}([0, \infty))$ which separates points and vanishes nowhere. Hence by the locally compact version of the Stone-Weierstrass theorem it is dense in $C_{0}([0, \infty))$ and consequently dense in $L^{1}(0, \infty)$. The claim now follows since $L^{1}(0, \infty)$ is the predual of $L^{\infty}(0, \infty)$ : let $F$ be such that all the functionals above are zero. Then for every $g \in L^{1}(0, \infty)$

$$
\int_{0}^{\infty} g(t) \exp (-\lambda t) F(t) \mathrm{d} t=0
$$

and consequently $e^{-\lambda t} F(t)=0$ for a.e. $t \in(0, \infty)$ hence $F=0$.

From the above Lemma we see that a minimal sequence $\left(\lambda_{n}\right)_{n}$ needed for Lerch's theorem is an arithmetic sequence of the form $\lambda_{n}=\lambda+n \zeta$ where $\lambda, \zeta>0$. For completeness we note that the converse implication regarding the Dafermos' criterium is not valid. More precisely, if a function satisfies the Dafermos' criterium (1.9) then it will not necessarily be chosen by our selection. This observation is based on the following result.

Lemma 5.4. Let $\mathcal{G} \subset L^{\infty}(0, \infty) \cap \mathrm{BV}_{\text {loc }}([0, \infty))$ and assume that $F \in \mathcal{G}$ satisfies $F \succ_{\mathcal{D}} G$ for every $G \in \mathcal{G}$. Then there exists $\lambda_{0}=\lambda_{0}(F, G)>0$ such that for all $\lambda \geqslant \lambda_{0}$ it holds

$$
\lambda \int_{0}^{\infty} \exp (-\lambda t) F(t) \mathrm{d} t>\lambda \int_{0}^{\infty} \exp (-\lambda t) G(t) \mathrm{d} t .
$$

Proof. We write

$$
\begin{aligned}
& \lambda \int_{0}^{\infty} \exp (-\lambda t)[F(t)-G(t)] \mathrm{d} t=\lambda \int_{0}^{\delta} \exp (-\lambda t)[F(t)-G(t)] \mathrm{d} t \\
& \quad+\lambda \int_{\delta}^{\infty} \exp (-\lambda t)[F(t)-G(t)] \mathrm{d} t \\
& \geqslant \lambda \exp (-\lambda \delta) \int_{0}^{\delta}[F(t)-G(t)] \mathrm{d} t-\left(\|F\|_{L^{\infty}}+\|G\|_{L^{\infty}}\right) \exp (-\lambda \delta) .
\end{aligned}
$$

Hence there exists $\lambda_{0}>0$ (depending on $F, G$ ) such that the right hand side is strictly positive provided $\lambda \geqslant \lambda_{0}$.

In other words, one would like to start the selection procedure given by an increasing sequence $\left(\lambda_{n}\right)_{n}$ with $\lambda_{1}$ as large as possible, in order to guarantee that the solution satisfying the Dafermos' criterium is selected. However, a uniform choice of $\lambda_{1}$ is not a priori known at the moment. 
Open Access This article is licensed under a Creative Commons Attribution 4.0 International License, which permits use, sharing, adaptation, distribution and reproduction in any medium or format, as long as you give appropriate credit to the original author(s) and the source, provide a link to the Creative Commons licence, and indicate if changes were made. The images or other third party material in this article are included in the article's Creative Commons licence, unless indicated otherwise in a credit line to the material. If material is not included in the article's Creative Commons licence and your intended use is not permitted by statutory regulation or exceeds the permitted use, you will need to obtain permission directly from the copyright holder. To view a copy of this licence, visit http://creativecommons.org/licenses/by/4.0/.

Publisher's Note Springer Nature remains neutral with regard to jurisdictional claims in published maps and institutional affiliations.

\section{References}

1. Benzoni-Gavage, S., Serre, D.: Multidimensional Hyperbolic Partial Differential Equations. First Order Systems and Applications. Oxford Mathematical Monographs. The Clarendon Press, Oxford (2007)

2. Breit, D., Feireisl, E., Hofmanová, M.: Solution semiflow to the isentropic Euler system. Arch. Ration. Mech. Anal., https://doi.org/10.1007/s00205-019-01420-6

3. Březina, J., Feireisl, E.: Maximal dissipation principle for the complete Euler system. Arxive Preprint Series, arXiv:1712.04761 (2017)

4. Březina, J., Feireisl, E.: Measure-valued solutions to the complete Euler system. Arxive Preprint Series, arXiv:1702.04878. J. Math. Soc. Japan 70, 1227-1245 (2018)

5. Cardona, J.E., Kapitanski, L.: Semiflow selection and Markov selection theorems. Arxive Preprint Series, arXiv: 1707.04778v1 (2017)

6. Chiodaroli, E., De Lellis, C., Kreml, O.: Global ill-posedness of the isentropic system of gas dynamics. Commun. Pure Appl. Math. 68(7), 1157-1190 (2015)

7. Chiodaroli, E., Kreml, O.: On the energy dissipation rate of solutions to the compressible isentropic Euler system. Arch. Ration. Mech. Anal. 214(3), 1019-1049 (2014)

8. Chiodaroli, E., Kreml, O., Mácha, V., Schwarzacher, S.: Non-uniqueness of admissible weak solutions to the compressible Euler equations with smooth initial data. Arxive Preprint Series, arXiv:1812.09917v1 (2019)

9. Dafermos, C.M.: The entropy rate admissibility criterion for solutions of hyperbolic conservation laws. J. Differ. Equ. 14, 202-212 (1973)

10. De Lellis, C., Székelyhidi Jr., L.: On admissibility criteria for weak solutions of the Euler equations. Arch. Ration. Mech. Anal. 195(1), 225-260 (2010)

11. Feireisl, E.: Maximal dissipation and well-posedness for the compressible Euler system. J. Math. Fluid Mech. 16(3), 447-461 (2014)

12. Feireisl, E., Klingenberg, C., Kreml, O., Markfelder, S.: On oscillatory solutions to the complete Euler system. Arxive Preprint Series, arXiv:1710.10918 (2017)

13. Hoff, D.: Local solutions of a compressible flow problem with Navier boundary conditions in general three-dimensional domains. SIAM J. Math. Anal. 44, 633-650 (2012)

14. Kato, T., Lai, C.Y.: Nonlinear evolution equations and the Euler flow. J. Funct. Anal. 56, 15-28 (1984)

15. Kröner, D., Zajaczkowski, W.M.: Measure-valued solutions of the Euler equations for ideal compressible polytropic fluids. Math. Methods Appl. Sci. 19(3), 235-252 (1996)

16. Krylov, N.V.: The selection of a Markov process from a Markov system of processes, and the construction of quasidiffusion processes. Izv. Akad. Nauk SSSR Ser. Mat. 37, 691-708 (1973)

17. Majda, A.: Compressible fluid flow and systems of conservationlaws in several space variables, volume 53 of Applied Mathematical Sciences. Springer, New York (1984)

18. Schochet, S.: The compressible Euler equations in a bounded domain: existence of solutions and the incompressible limit. Commun. Math. Phys. 104, 49-75 (1986)

19. Smoller, J.: Shock Waves and Reaction-Diffusion Equations. Springer, New York (1967)

20. Stroock, D.W., Varadhan, S.R.S.: Multidimensional Diffusion Processes. Classics in Mathematics. Springer, Berlin (2006). Reprint of the 1997 edition

Communicated by C. De Lellis 